\begin{tabular}{|c|c|}
\hline Citation & $\begin{array}{l}\text { Mosquera-Sánchez, J. A.; Sarrazin, M.; Janssens, K.; de Oliveira, L. P. R.; } \\
\text { Desmet, W., (2018). } \\
\text { Multiple target sound quality balance for hybrid electric powertrain } \\
\text { noise. } \\
\text { Mechanical Systems and Signal Processing, v. 99, 478-503. }\end{array}$ \\
\hline Archived version & $\begin{array}{l}\text { Author manuscript: the content is identical to the content of the published } \\
\text { paper, but without the final typesetting by the publisher. }\end{array}$ \\
\hline Published version & https://doi.org/10.1016/j.ymssp.2017.06.034 \\
\hline Journal homepage & https://www.journals.elsevier.com/mechanical-systems-and-signal-processing \\
\hline Author contact & $\begin{array}{l}\text { mosquerax@gmail.com } \\
+55 \text { (16) } 99742 \text { - } 1291\end{array}$ \\
\hline IR & \\
\hline
\end{tabular}

(article begins on next page) 


\title{
Multiple target sound quality balance for hybrid electric powertrain noise
}

\author{
Mosquera-Sánchez, J. A. ${ }^{\mathrm{a}, \mathrm{b}, *}$, Sarrazin, M. ${ }^{\mathrm{c}}$, Janssens, K. ${ }^{\mathrm{c}}$, de Oliveira, L. P. R. ${ }^{\mathrm{a}}$, Desmet, W. ${ }^{\mathrm{b}, \mathrm{d}}$ \\ ${ }^{a}$ Department of Mechanical Engineering, São Carlos School of Engineering, University of São Paulo, Av. Trabalhador Sancarlense 400, \\ 13566-590 São Carlos (SP), Brazil \\ ${ }^{b}$ Department of Mechanical Engineering, PMA Division, KU Leuven, Celestijnenlaan 300, 3001 Heverlee, Belgium \\ ${ }^{c}$ Siemens Industry Software NV., Interleuvenlaan 68, B-3001 Leuven, Belgium \\ ${ }^{d}$ Member of Flanders Make, Belgium
}

\begin{abstract}
The integration of the electric motor to the powertrain in hybrid electric vehicles (HEVs) presents acoustic stimuli that elicit new perceptions. The large number of spectral components, as well as the wider bandwidth of this sort of noises, pose new challenges to current noise, vibration and harshness (NVH) approaches. This paper presents a framework for enhancing the sound quality (SQ) of the hybrid electric powertrain noise perceived inside the passenger compartment. Compared with current active sound quality control (ASQC) schemes, where the SQ improvement is just an effect of the control actions, the proposed technique features an optimization stage, which enables the NVH specialist to actively implement the amplitude balance of the tones that better fits into the auditory expectations. Since Loudness, Roughness, Sharpness and Tonality are the most relevant SQ metrics for interior HEV noise, they are used as performance metrics in the concurrent optimization analysis, which, eventually, drives the control design method. Thus, multichannel active sound profiling systems that feature cross-channel compensation schemes are guided by the multi-objective optimization stage, by means of optimal sets of amplitude gain factors that can be implemented at each single sensor location, while minimizing cross-channel effects that can either degrade the original SQ condition, or even hinder the implementation of independent SQ targets. The proposed framework is verified experimentally, with realistic stationary hybrid electric powertrain noise, showing SQ enhancement for multiple locations within a scaled vehicle mock-up. The results show total success rates in excess of $90 \%$, which indicate that the proposed method is promising, not only for the improvement of the SQ of HEV noise, but also for a variety of periodic disturbances with similar features.
\end{abstract}

Keywords: Hybrid electric vehicle, multichannel active sound quality control, multi-objective sound quality optimization, periodic disturbance, sound quality

\section{Introduction}

Economical and environmental aspects compel the automotive industry to make a turn towards eco-friendly and sustainable machine and system design [1-8]. In this direction, the possibility of electrifying vehicle powertrains has become one of the main subjects of research in the industry, on the basis that the electric motor (EM), which arguably exhibits better performance than the internal-combustion engine (ICE), is a cleaner source of motive power [2, 6, 9-12]. Attributes, such as its high performance, still at low rotation speeds, its zero emission operation, as well as its reduced manufacturing costs, make the EM the preferred choice among the available propulsion technologies. However, since the duration of power supply for EMs is currently seen not to endure long journeys, diverse levels of powertrain electrification have been proposed $[1,6,13-16]$. While the electric vehicle (EV) is intended for short trips,

\footnotetext{
* Corresponding author.

Email addresses: jamosquera@usp.br (Mosquera-Sánchez, J. A.), jaime .mosquera@kuleuven .be (Mosquera-Sánchez, J. A.), mathieu. sarrazin@siemens . com (Sarrazin, M.), karl. janssens@siemens. com (Janssens, K.), leopro@sc.usp. br (de Oliveira, L. P. R.), wim. desmet@kuleuven. be (Desmet, W.)

URL: http://www.eesc.usp.br/labdin/leopoldo.htm (de Oliveira, L. P. R.)
} 
e.g. operation in cities, the HEV benefits from integrating ICEs to its powertrain for extending its autonomy. Even so, in spite of the cost of HEVs as compared to standard ICE cars [1,9,13], they did have succeeded in positioning themselves as the preferred customer choice amongst electrified cars [12, 17]. The extended autonomy is mostly acknowledged [5], while still relying on the use of clean sources that provide motive power [11].

Vehicles with hybrid powertrains typically comprise of an important number of parts that operate in different modes, which depend on the current rotation speed, load, breaking and/or recharging regimes [6, 13, 18]. Some operation modes even demand the parts to co-operate amongst them [19, 20]. As one would intuitively reason, the interaction amongst those subsystems results in sounds that are substantially different from that of the ICE itself $[20,21]$. In particular, the power inverter, which is the part that commands the speed regime of the EM, generates new audible components that are multiples of the switching frequency of the driver. The presence of new audible components in the noise will arguably elicit other than the impressions ICE-propelled vehicles typically evoke [14]. For the ICE-propelled vehicle user who is willing to take a step towards HEVs, it will not be an easy auditory experience to get used to the fact of having such a large amount of harmonics. Another novel but unexpected HEV sound feature is the fact that the spectral lines in the auditory stimulus spread in a larger bandwidth [1]. These NVH aspects may prevent HEVs to be more accepted by customers [8], even when its environmental features are proven to be better than the ones of the ICE-propelled vehicle.

The relationship between the physical parameters of technical sounds and the auditory perception is also currently one of the spotlights of the automotive industry [22]. The possibility for tailoring the residual powertrain noise to provide the driver and passengers with relevant auditory information on the operation regime of the vehicle [23-27], or even for enhancing customer perceptions [28, 29] of, e.g. vehicle quality, powerfulness, sportiveness, luxury, reliability quietness, among others [30], also enables the manufacturers to boost their products with unique and distinctive sound marks [31-36]. At that point, SQ techniques come in handy for supporting auditory-oriented procedures, since they bridge the gap between objective vibro-acoustics and the human auditory response, by means of models that quantify auditory perceptions. Whereas it is still possible to cope with the unusual amount of harmonics and spectral crossings amongst them in the hybrid electric powertrain noise, by simply following SQ techniques applied for ICE noise, the fact that its spectrum spreads over a wider bandwidth, sometimes in excess of $4 \mathrm{kHz}$, does demand the inclusion of more auditory dimensions to the problem, namely other than the widely used low-frequency Loudness [28, 34, 36-43] and Roughness [28, 34-39, 42, 44-46]. In fact, some recent works on NVH aspects of HEVs [1, 6, 47] put forward the need for developing SQ approaches that face tonal, narrowband and sharp sounds, which are the novel high-frequency occurrences in the noise that come with integration of the EM in the powertrain. Then, high-frequency SQ metrics, namely Sharpness [20, 28, 34, 37, 38, 40, 42] and Tonality [20, 28, 41, 46, 48], should be accounted for when objectively diagnosing -and controlling- the SQ of hybrid electric powertrain noise.

In this line of thought, this paper presents a framework to enhance the auditory perception of the hybrid electric powertrain noise, which copes with the possibility of adapting a sound field towards concurrently optimal SQ targets to each of the four auditory dimensions associated to the sort of noises of concern: Loudness, Roughness, Sharpness and Tonality. In particular, the noise emitted by hybrid electric powertrains, which is structure borne and measured at a number of relevant locations in the vehicle cabin, is tackled through an innovative multichannel distributed algorithm that delivers independent-zone active sound profiling [49]. Contrary to current active noise equalization (ANE) [24, 25, 27-29] and active sound quality control (ASQC) strategies [26, 32, 33, 36, 39, 50, 51], where the improvement of the soundscape comes as a mere effect of applying a few operation modes, e.g. cancellation, reduction, or amplification, the proposed methodology features a multi-objective optimization stage, which provides the NVH specialist with a variety of optimal solutions, given in terms of amplitude levels that should be attained in the sound, so as to provide the passengers with the exact, desired SQ levels. In this form, amplitude gains of ANE/ASQC schemes in between the widely used $0.0-2.0$, which correspond to the cancellation and amplification operation modes, respectively, including the so-called preservation, or inactive mode, i.e. $g=1.0$, become relevant in the proposed framework.

In general terms, the proposed framework for the SQ enhancement of hybrid electric powertrain noise involves four key steps: (i) the diagnosis of the SQ, as measured in the passengers compartment; (ii) the search for enhanced conditions for the sound; (iii) the real-time implementation of the SQ targets via SISO/MIMO active sound quality control schemes; and (iv) the assessment of the controlled sound, in order to verify whether the implemented control tasks did effectively improve its qualities, as prescribed in the optimization stage. The effectiveness of the proposed framework is experimentally demonstrated in a 1:3-scaled vehicle mock-up, instrumented with four sensor/actuator 
pairs (SAPs) at locations that emulate driver, co-driver and rear passengers. A stationary periodic sound, whose timefrequency pattern has been extracted from a real hybrid electric powertrain emission, is used to excite the mock-up, while ten different optimal solutions have been tested on the sound, for each of the four targeted locations. The controlled sound is eventually assessed with time-domain SQ models, which enables the comparison between optimized and attained SQ scores. Mean success rates obtained from comparing optimized and attained SQ scores above $90 \%$ demonstrate the validity of the proposed methodology.

This paper has been organized as follows: Section 2 introduces the proposed multi-objective SQ optimization procedure, together with discussions on time- and frequency-domain SQ metrics and algorithms, as well as sensitivity analyses that allow the reduction of the complexity of the multi-objective optimization (MOO) step. Section 3 describes a frequency-domain multichannel active sound profiling algorithm [49], which allows the implementation of the prescribed SQ targets from the MOO step, at each of the targeted locations within the experimental cavity. Section 4 presents experimental results. Section 5 concludes this paper with remarks and future work on this research.

\section{Multi-objective sound quality optimization of periodic disturbances}

Current approaches for the design and analysis of the SQ of a product can be framed into two lines of thought, according to quality management theories [52,53], as follows: (i) the manufacturing-based approach, which deals with the degree to which a product conforms to a design or specification; or (ii) the user-based approach, which is concerned with the degree to which a product satisfies the wants of a specific consumer. While most of the NVH studies in passenger cars are oriented towards reducing disturbance levels, or even for complying with product standards and directives, recent papers have put forward the need for innovative vehicle design procedures that truly aims at satisfying the expectations of -specific- customers $[39,46,54,55]$. Although intuitively the reduction of noise and/or vibration levels should always be perceived as good, human related perceptions might result negatively affected, once psycho-acoustic issues related with masking, pitch, equalization, etc. are not foreseen. Therefore, NVH refinement can only be achieved if, besides level reduction (or equalization), all the relevant psycho-acoustic features are taken into account. The open question at that point is related to the correlation between the modifications to the powertrain emitted tonal components and the enhancement of its sound qualities, as perceived by the passengers.

This section devotes to describe a multi-objective optimization (MOO) problem, posed for guiding active control actions towards the enhancement of the sound qualities of the noise emitted by the powertrain in HEVs. Prior to detail the proposed MOO problem, the underlying concepts behind each of the associated SQ metrics are briefly described.

\subsection{Sound quality metrics}

Psycho-acoustic models for Loudness [56, 57], Roughness [35, 56, 58], Sharpness [56] and Tonality [41, 48, 59] have been proposed for estimating their subjective constructions. These models take the time series of an acoustic emission as the input. In this regard, long measurements of the acoustic emission of rotating machines at stationary regimes can be thought as if they were periodic extensions of a single cycle. Thus, all the psycho-acoustic information could be inferred from just one signal cycle, as long as the fundamental period of the noise remains constant during uptime. This suggests that frequency-domain psycho-acoustic models can be a feasible and computationally efficient way to assess the SQ of such sounds. In this way, the frequency-domain versions of those algorithms can be used for further optimization routines. Then, this section describes calculations related to both time- and frequency-domain approaches for estimating Loudness, Roughness, Sharpness and Tonality of the acoustic emission of rotating machines, which is the case of the powertrain noise in either electric or hybrid electric vehicles.

\subsubsection{Loudness}

Psycho-acoustic Loudness is the model that characterizes the auditory perception of the sound intensity or volume. The so-called Zwicker-Loudness model [56] accounts for individual contributions to the total spectrum, discriminated by critical bands, as well as for masking effects. One sone is the sensation elucidated by the $1 \mathrm{kHz}, 40 \mathrm{~dB}_{S P L}$ signal, i.e. by a 40 phon signal. Time- and frequency-domain algorithms for calculating Loudness are summarized in Fig. 1.

As it can be seen, the frequency-domain implementation saves the FFT step in each iteration, as it is based on the already available frequency-domain data of the disturbance. This represents a significant enhancement in efficiency for the method, once that the controller action will also be dealt with in the frequency domain, by altering the amplitude and/or relative-phase of the tones, avoiding FFT and IFFT calculations for each configuration analyzed. 


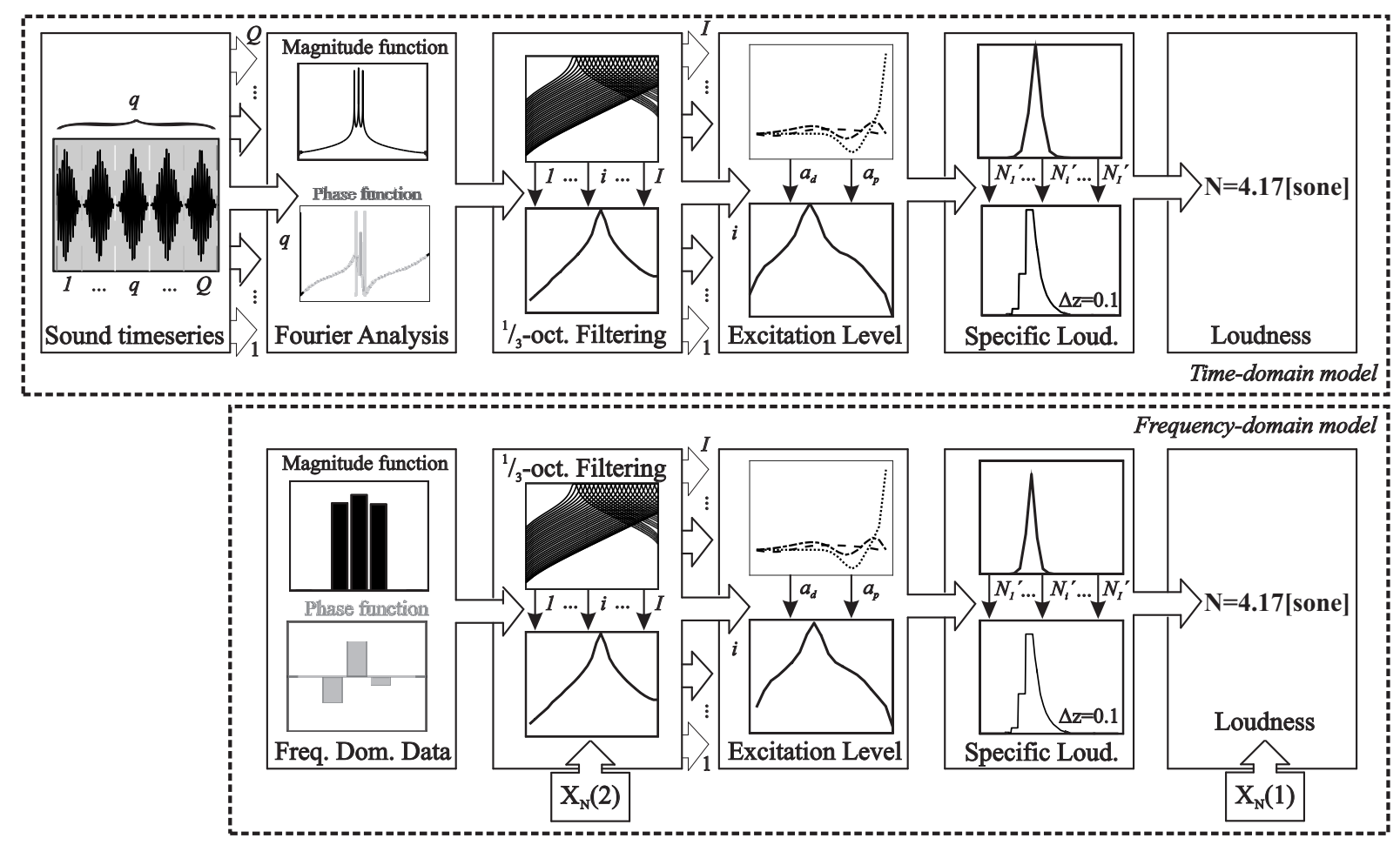

Figure 1: Block diagrams of time- and frequency-domain Loudness models.

\subsubsection{Sharpness}

According to Fastl and Zwicker [56], psycho-acoustic Sharpness is the model that describes the auditory sensation related to the sound density. Although this metric has not been standardized, psycho-acoustic Sharpness is used for assessing the SQ of technical sounds with high-frequency content [28]. Sharpness is measured in acum, which is defined as the perception elucidated by the noise filtered within a 1-critical-band-wide filter, centered at $1 \mathrm{kHz}$, and whose root-mean-square (RMS) value equals $80 \mathrm{~dB}_{S P L}$. Since psycho-acoustic Sharpness is a model derived from that of Loudness, the corresponding calculations are straightforward to grasp. Figure 2 illustrates block diagrams of both time- and frequency-domain algorithms for the calculation of this metric.

As discussed previously, the use of a frequency-domain algorithm here will also improve the computational efficiency for the optimization step, by skipping Fourier analyses for each configuration step.

\subsubsection{Tonality}

Psycho-acoustic Tonality is the model that extracts the tonal characteristic of a sound. Tonality is strongly related to the pitch of sounds. The mathematical model proposed by Terhardt has been studied by some authors, e.g. Shin et al. [41], Zhang and Shrestha [48], Shin et al. [59], which has been adapted for meeting specific soundscapes: internal combustion (IC) engine noises, laser printer noises, among others. Since temporal effects of sounds can be better described by metrics such as Roughness $[41,46]$, the place theory for approaching the pitch perception, which assumes that the human auditory system discriminates sounds by frequencies, seems to suffice for the task of characterizing the Tonality of periodic sounds. An unit for Tonality has been defined by Shin et al. [41], whose adjusted the so-called spectral pitch strength of a $1 \mathrm{kHz}, 80 \mathrm{~dB}_{S P L}$ pure-sinusoid signal to have unit value. Figure 3 shows the calculation flows of the time- and frequency-domain Tonality algorithms, which again shows the benefit of the frequency-domain method over the more traditional time-domain algorithm.

\subsubsection{Roughness}

Psycho-acoustic Roughness is the model that quantify the perception of amplitude fluctuations in the range between $20-200 \mathrm{~Hz}$ [60]. Auditory Roughness is quantified in asper, in which 1 asper is the perception elucidated by 


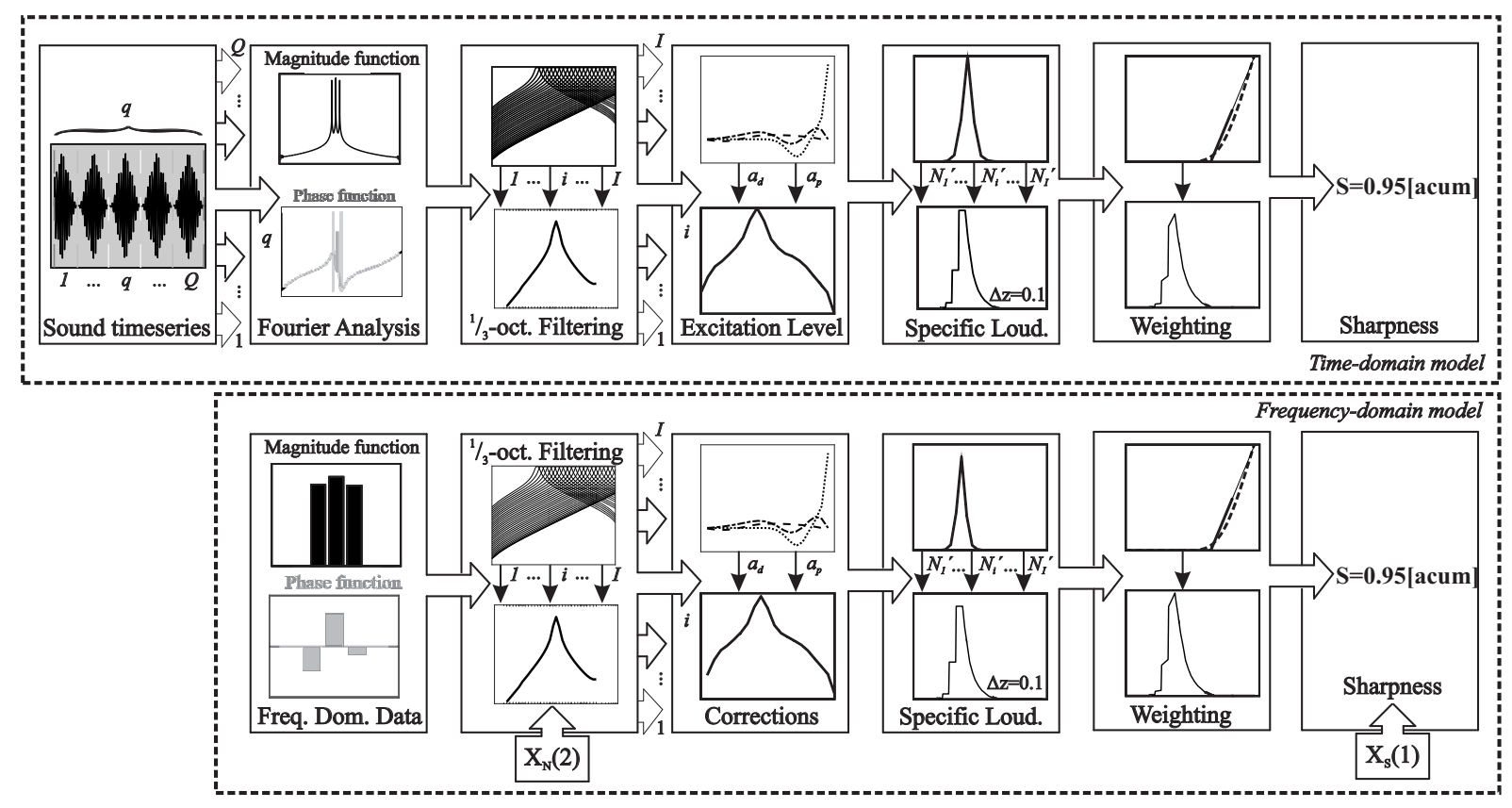

Figure 2: Block diagrams of time- and frequency-domain Sharpness models.

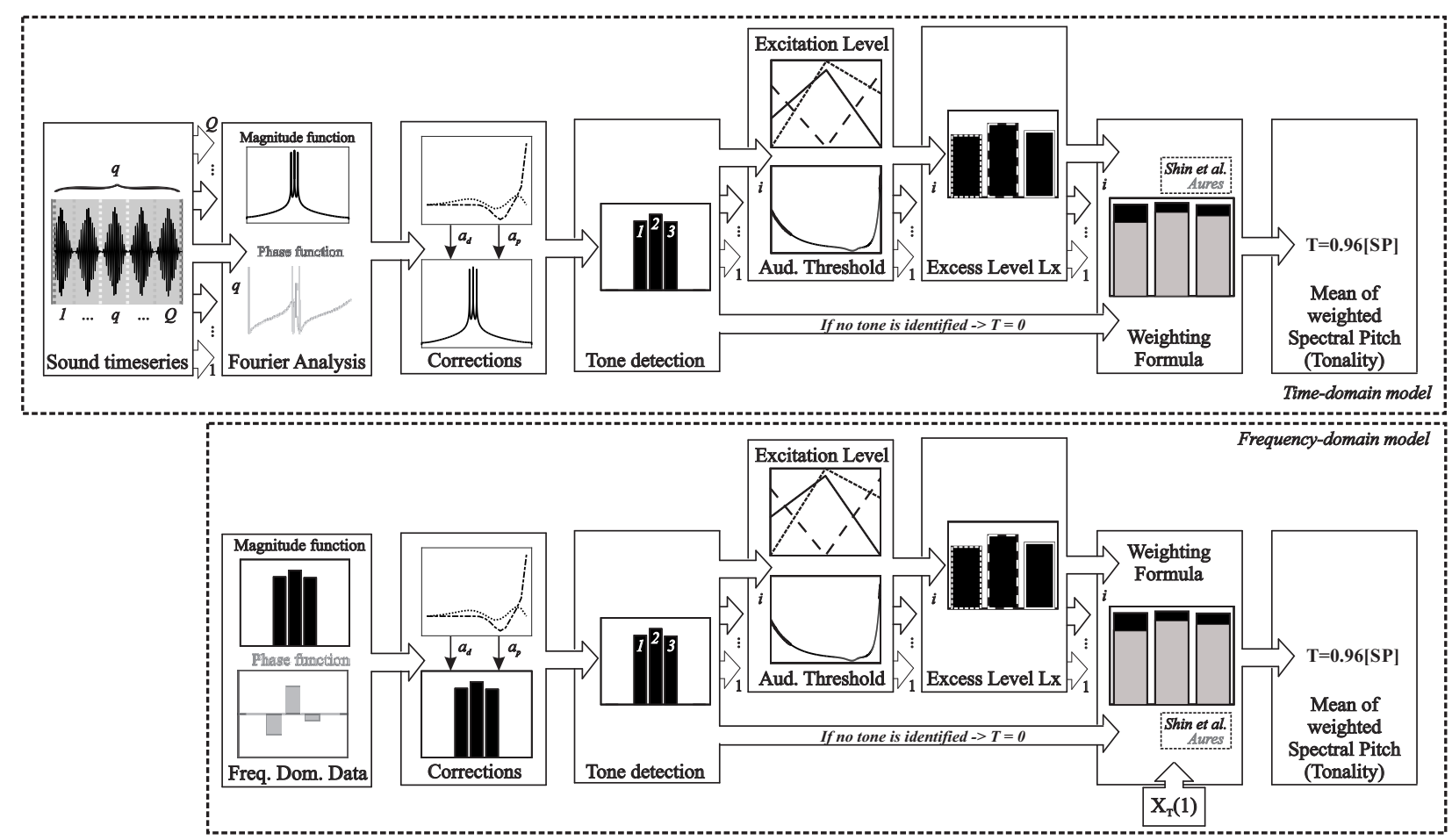

Figure 3: Block diagrams of time- and frequency-domain Tonality models.

the $1 \mathrm{kHz}, 60 \mathrm{~dB}_{S P L}$ sound that is $100 \%$ amplitude-modulated by a pure-sinusoid signal whose frequency is $70 \mathrm{~Hz}$ [56]. Auditory Roughness is a largely studied psycho-acoustic metric. However, no general consensus exists on a standardized model that quantifies this perception. 
The semantic interpretations that some authors have conferred to this psycho-acoustic sensation are, in some sense, contradictory. On the one hand, de Baene et al. [60] denote auditory Roughness as an impure or unpleasant sound quality. Daniel and Weber [58] argue that Roughness in sounds is seen to reduce its sensory pleasantness. Wang et al. [35] coin terms such as rattle and creak to auditory stimuli that elucidate Roughness. On the other hand, NVH specialists in the automotive industry do take advantage of this perception for inducing, or even enhancing, sensations like sportiveness or powerfulness of ICE noises perceived at the interior of passenger cars [28, 30, 33, 36, 38, 61].

Time-domain Roughness models proposed by Daniel and Weber [58], Hoeldrich and Pflueger [62] and Wang et al. [35] have been studied in detail. The incorporation of relative-phase influences, reported on by Pressnitzer and McAdams [63] and confirmed by Kohlrausch et al. [64], have also been taken into account. A computationally optimized algorithm for calculating Roughness of ICE noise, proposed by Janssens et al. [44], has also been studied in detail, once that this is the first frequency-domain SQ metric reported on the specialized literature. Figure 4 illustrates both time- and frequency-domain approaches for calculating this metric.

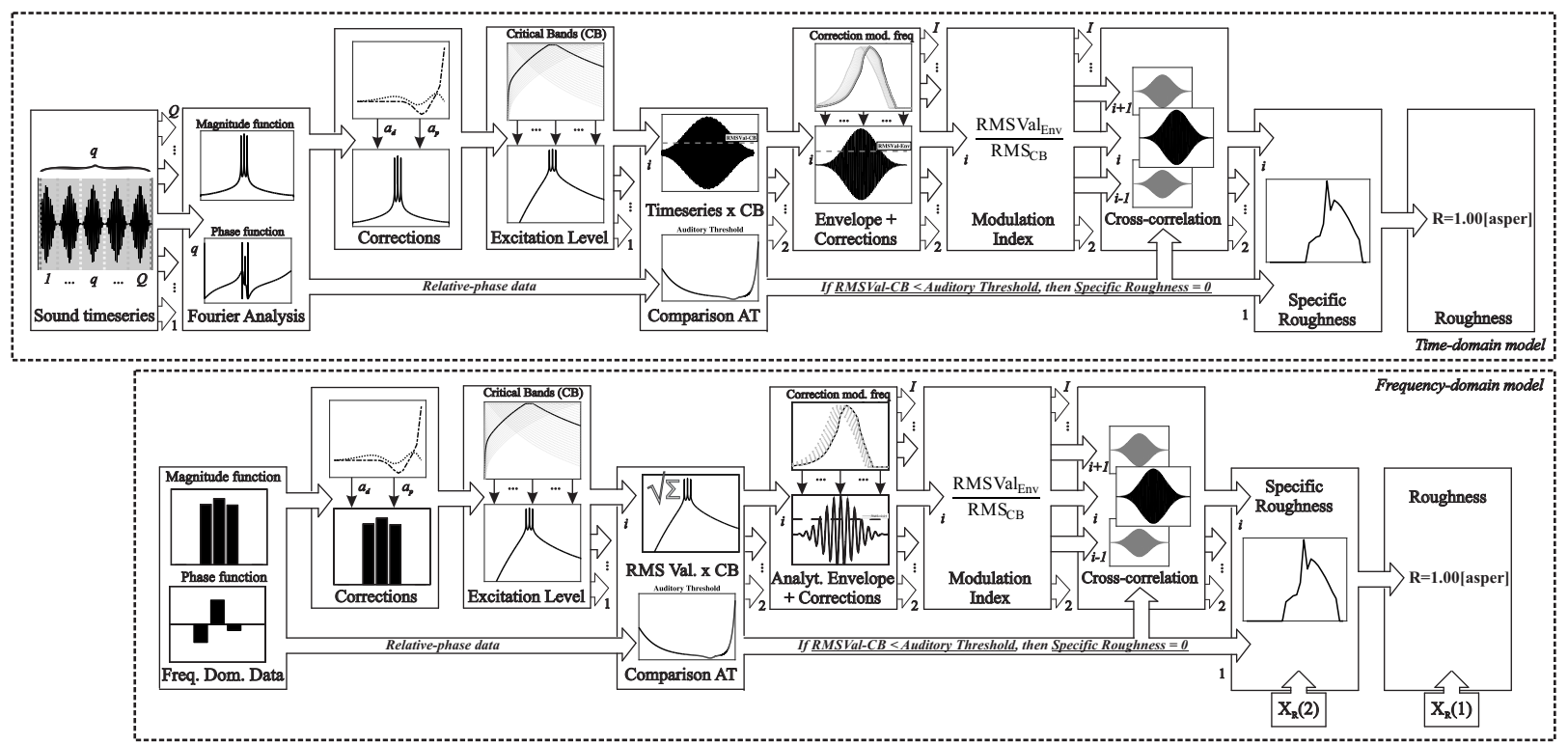

Figure 4: Block diagrams of time- and frequency-domain Roughness models.

\subsection{Multi-objective sound quality optimization}

On the basis of the aforementioned SQ models, the specialized literature shows a number of studies that attempt to describe and predict the SQ of a noise with a single scalar value. Their level of complexity ranges from linear formulations, e.g. the weighted sum of metrics and linear regressions [22, 28, 34, 37, 40-42, 45, 65, 66], to complex non-linear ones, e.g. the Sensory Pleasantness model [56], as well as neural network-based formulations [38, 67-71]. However, in the light of the variety of opinions that is encountered on how a given machine or system should sound, the qualification of its byproduct noise through a single scalar value, or an adjective (good, pleasant, smooth, sporty, among others), can be imprecise and rather difficult to reproduce.

Recalling that the main objective of the present methodology is to propose active control systems that enables the SQ enhancement of HEV noise, the arrangement of the SQ metrics in a vector form is used and its optimization is worked out in a multi-objective fashion. The purpose of this multi-objective optimization (MOO) problem is to provide such systems with a target, which is based on the physical properties of the disturbance. Therefore, a mapping of such physical quantities (e.g. tone levels) and the SQ perceptions becomes necessary.

The multi-objective SQ optimization problem for periodic disturbances is posed as follows:

$$
\begin{array}{rll}
\min _{\mathbf{a}} \mathbf{Y} & =\min \left(\mathbf{F}_{i}(\mathbf{a})\right) ; & i=1, \ldots, Q ; \\
\text { subject to } & : \quad a_{j}^{(L)} \leqslant a_{j} \leqslant a_{j}^{(U)} ; \quad j=1, \ldots, V ; \quad V \leqslant R
\end{array}
$$


where $\mathbf{a} \in \mathbb{R}^{V}$ is the vector composed of the amplitude values of the tones that belong to the decision space, where $V \leqslant R$ is the amount of tones that has been associated to the MOO problem; each of the $\mathbf{F}_{i} \rightarrow \mathbf{Y} \in \mathbb{R}^{Q}$ functions are the associated SQ dimensions to the sort of noises of concern, which belong to the feature [69] or objective space; and superscripts $\{L, U\}$ stand for lower and upper bounds given for each of the $V$ harmonics in the decision variable set. Eq. 1 describes an optimization problem of a set of SQ metrics, with constraints on the amplitude values that each tone in the decision space set can take.

The powertrain noise in HEVs has a large amount of tones, which would jeopardize any attempts on assessing a broad combination of possible solutions for the enhancement of its SQ. It would also be unfeasible to require an active control system to deal with such an amount of variables. Therefore, at this point it seems reasonable to work with a reduced set of tones, both in this stage, as well as in the practical implementation of the active sound quality control (ASQC) stage. The underlying hypothesis is that one can find a reduced set of tones that most drastically affect each SQ perception. Hence, sensitivity analyses on the SQ metrics are performed, in order to render the optimization stage feasible.

\subsubsection{Decision space: sensitivity analyses}

One of the main features in human perception that leads to the conclusion that not all of the narrowband components of a sound are relevant to noise control, is that of masking. This mechanism depends on the relative level and frequency gap between two (or more) tones, which may result in the dominant one to act as a masker, i.e. to actually block the aural contribution of the neighboring sound. In this way, any reduction of the masked orders, although may show as SPL reduction, will have no effect on the perception of the sound. This feature alone, which affects at least Loudness and Sharpness, advocates for the sensitivity analysis, yet other phenomena could be listed, such as the filtering of the peripheral auditory system that happens in the outer, middle and inner ear; the weighting of tonal components that happens into the central auditory system; particularities observed when exposed to modulated disturbances, among others.

In this way, each of the SQ metrics associated to the MOO is proposed to undergo a sensitivity analysis, which will highlight the spectral components that truly drive the sound under study to noticeable changes in perception. To this concern, the so-called Elementary Effects (EE) method is used, which relies on One-factor-at-a-time-based input variations, investigated in previous research [50], in order to provide two sensitivity measures: (i) the measure $\boldsymbol{\mu}$ that describes the relevance of the inputs, and (ii) the measure $\boldsymbol{\sigma}$ that relates to non-linear effects and/or interactions amongst variables [72]. These measures are determined as follows:

$$
\mu_{j}=\frac{1}{R} \sum_{k=1}^{R} d_{j}\left(a_{k}\right) ; \quad \sigma_{j}=\sqrt{\frac{1}{R-1} \sum_{k=1}^{R}\left[d_{j}\left(a_{k}\right)-\mu_{j}\right]^{2}},
$$

where:

$$
d_{j}(\mathbf{a})=\frac{F_{i}\left(a_{1}, \ldots, a_{j-1}, a_{j}+\Delta, a_{j+1}, \ldots, a_{R}\right)-F_{i}(\mathbf{a})}{\Delta}
$$

is the so-called elementary effect the $j^{\text {th }}$ input variable, i.e. the $j^{\text {th }}$ tone, causes on the $i^{\text {th }}$ cost function, i.e. the $i^{\text {th }}$ SQ metric, by keeping all of the other variables at their nominal amplitude values. Each of the input variables is assumed to vary across $p$ levels in the decision space. $F_{i}(\mathbf{a})$ stands for the nominal value for $F_{i}$, i.e. the function value at the original condition of the amplitudes of the primary sound, a. The EE method should be carried out for the entire set of tones, $R$, in order to investigate all the effects on each SQ model, together with possible interactions amongst the tones. Equations 2 and 3 can be carried out with $\Delta=1 /(p-1)$, and $p \in \mathbb{N}$, i.e. a positive integer number of levels, in order to alleviate for the computational demands of this procedure.

Figure 5(a) illustrates the implementation of the EE method, when mapping the sensitivity of 3 tones to a certain SQ metric. The range in the EE method targeting noise control would only consider values between the nominal amplitude and a lower bound (e.g. maximum noise reduction). However, the present implementation foresees active noise equalizations with four modes of operation: cancellation, reduction, preservation (or inactive) and amplification, hence the used range is $\left[0, a_{j}+6\right] \mathrm{dB}_{S P L}$, or $\left[20 e^{-06}, 2 a_{j}\right] \mathrm{Pa}$. The amplification mode could offer the masked components the "opportunity" to become relevant, as their amplitude could reach up to twice their original level. With the bounds defined for each variable, the amplitudes of the tones in the sound are mapped into a hypercube, and $\Delta$ 
is computed by defining a number of levels, which reads $p=5$ for the example in Fig. 5(a). It also illustrates the augmented cube that would result if just three tones were taken into account. Nevertheless, it has to be recalled that all of the tones in the primary sound should be considered. Thus, each of the $\Delta$ values are brought back to the amplitude domain for calculating the trajectory, i.e. the amplitude vector, of each variable. Next, Eq. 3 is calculated at each increment of $\Delta$. As soon as this procedure is repeated for the $R$ tones, the measures $\mu$ and $\delta$ can be obtained. The tone(s) that cause the maximum $\mu$ values can be regarded as the most involved ones in producing large variations on each single SQ metric.

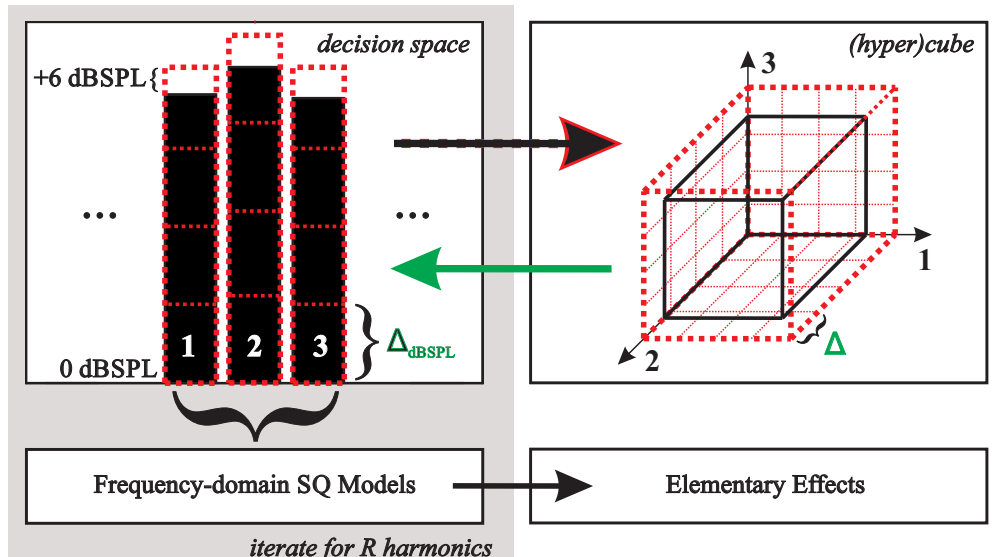

(a)

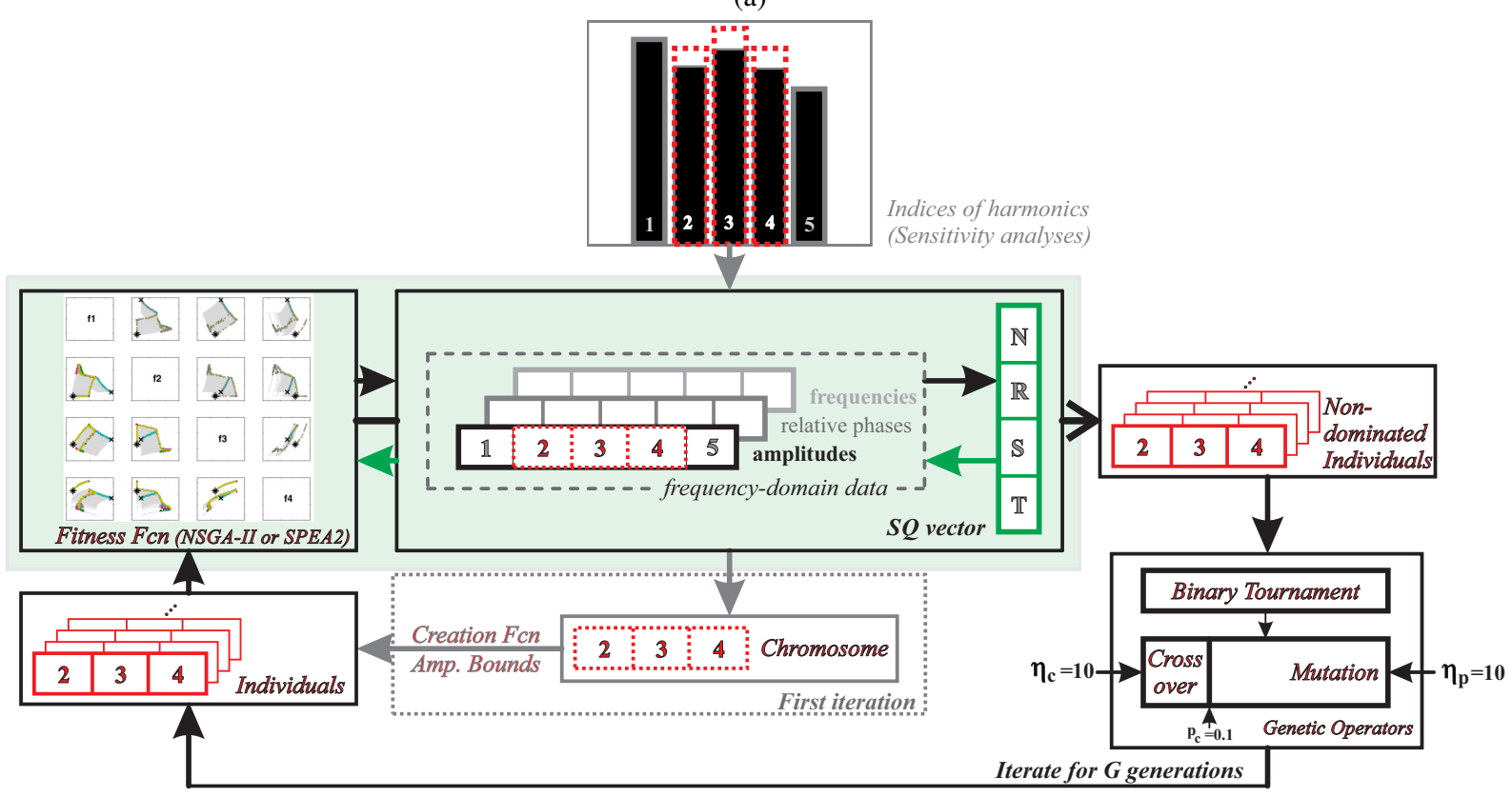

(b)

Figure 5: (a) flow chart of sensitivity analyses based on the elementary effects method; (b) flow chart of the multi-objective optimization based on evolutionary solvers.

\subsubsection{Objective space: multi-objective evolutionary solvers}

As presented early, Loudness, Roughness, Sharpness and Tonality metrics have been associated with a wide sort of technical noises. While Loudness and Roughness are good SQ descriptors for low- and mid-frequency 
noise, Loudness, Sharpness and Tonality are used for sounds with high-frequency content. Then, it seems reasonable to quantify the SQ of the hybrid electric powertrain noise by using these metrics, once this sort of sounds spread into a wide bandwidth, usually from lower than $50 \mathrm{~Hz}$, up to $10 \mathrm{kHz}$. This leads to rewrite $\mathbf{Y}$ in Eq. 1 as $\mathbf{F}=[N(\mathbf{a}), R(\mathbf{a}), S(\mathbf{a}), T(\mathbf{a})]^{T}$, i.e. the SQ vector associated to the hybrid electric powertrain noise consists of a fourth-dimensional objective space.

The task of optimizing vectors is currently approached by using evolutionary algorithms, since they implement special strategies for handling the cost function into multi-dimensional objective spaces [73]. Moreover, as long as the functions arranged in a vector are conflicting, i.e. the improvement of one single objective comes at the expense of the remaining ones, it is expected that a set of diverse solutions, rather than a single one, is obtained from the concurrent optimization [74]. This set of diverse solutions is known along the literature as the Pareto front, or compromise frontier. The attainment of the Pareto fronts in the proposed MOO problem has been investigated by using two multi-objective evolutionary solvers, namely the Non-dominated Sorting Genetic Algorithm (NSGA-II) [75] and the Strength Pareto Genetic Algorithm (SPEA2) [76, 77]. Previous research has shown that any of these multi-objective evolutionary solvers is able to converge to the optimal Pareto front in the problem of concern [78], so the NSGA-II outcomes will only be discussed along this paper.

While the operation of any of the chosen evolutionary solvers follow the typical data flow of the genetic algorithm, i.e. encoding of variables, definition of population size and number of generations, evaluation of the fitness function and implementation of genetic operators (binary tournament, simulated binary crossover, SBX and polynomial mutation), the assessment of the fitness function is carried out by each solver in an unique fashion. Whereas the NSGA-II bases its operation on the calculation of the crowding distance measure [75], the SPEA2 assess the fitness function by calculating density estimates of the non-dominated solutions, based on the $k^{\text {th }}$ nearest neighbor method [77].

Figure 5(b) illustrates an application example of the proposed MOO for the enhancement of the SQ of hybrid powertrain noise. It can be observed that, provided that a reduced set of tones in the primary sound has been defined for driving the MOO, the size of the population turns out to be computationally affordable. Moreover, the block diagram in the cited Figure clearly illustrates that each evaluation of the SQ vector, i.e. the cost function, takes into account the entire amplitude and relative-phase vectors that correspond to each tone in the primary sound. Then, the computational routines just need to load into memory the indices of the targeted tones, as well as the original frequency-domain data of the primary sound. Thus, the amplitude values from each individual at the current iteration are replaced into the entire vector of amplitudes, in order to evaluate the SQ vector, with the frequency-domain methods, along generations.

\subsection{Remarks}

This section has introduced a MOO problem, especially devised for investigating for better equalizations of the magnitude function of a given periodic disturbance, such that the associated SQ perceptions are enhanced. Four SQ dimensions have been associated to the noise emitted by the powertrain in HEVs, namely Loudness, Roughness, Sharpness and Tonality, since they cover the bandwidth the noises of concern typically spread into. Moreover, since this sort of noise is composed of a number of tones, sensitivity analyses based on the EE method have been proposed, in an attempt for reducing the level of complexity of the design space. Multi-objective evolutionary algorithms such as the NSGA-II and the SPEA2 have been investigated in the search for the optimal Pareto fronts. The optimal Pareto fronts provide a set of amplitude values for each of the decision variables, such that the four SQ perceptions can be driven towards enhanced scores. Since no analytical solution is known for the optimization problem at hand, the verification of the attainment of the true Pareto fronts has been carried out through comparisons amongst the outcomes from both evolutionary solvers, which are at their turn contrasted with results from exhaustive search procedures. This will be shown and discussed in the section that presents the experimental results, i.e. Section 4.

\section{Multichannel active sound quality control}

In the context of automotive applications, the sound emitted by the powertrain is propagated through the vibroacoustic system, thus reaching relevant locations within the passenger compartment. As it can be expected, the dynamics of the system modifies the original time/frequency pattern of the auditory stimulus, such that both of the magnitude and relative-phase spectral functions of the noise perceived are reshaped. Whereas in both free field 
radiation and enclosures the system delay has an important role in defining the relative-phase function, the amplitude function is strongly affected by the cavity mode shapes and damping. Moreover, the location of the error sensor(s), or listener(s), play(s) an important role in the perception of the noise, since at some locations, modal features may either reinforce or mitigate some harmonics originally present in the source. Hence, it is expected that the perception of the primary sound differs from one location to another. These effects, which are particularly relevant in cavities with low damping, require the proposed methodology for approaching the SQ to be carried out for each targeted location. As a result, it is possible that the sensitivity analysis will highlight a different set of harmonics to be controlled at each location. It is worth noticing that, if a certain harmonic needs to be changed only at one location, it does not mean it should not be controlled at the other targeted locations; it does mean that it should be preserved at those places. Therefore, a multichannel controller devised for implementing optimal SQ targets will eventually have to manage all the harmonics highlighted by each of the sensitivity analysis, in some locations by adjusting the gain factors according to the SQ optimization and, at others, by preserving its original amplitude.

As soon as the multichannel control scheme to be used delivers free-of-interference active sound profiling actions, the proposed MOO framework can be independently executed for each of the targeted locations in the system. To this end, a set of multichannel active periodic sound profiling algorithms has been proposed in previous research $[49,51]$, which features an innovative scheme for compensating for cross-channel interferences. The main concept behind the proposed strategy relies on the fact that the acoustic coupling terms, which are neglected from the puredecentralized multichannel formulation due to computational cost reasons, should be taken into account. While the acoustic coupling amongst channels is indeed taken into account in the centralized multichannel formulation, the proposed distributed control schemes also account for the coupling, in such a form that independent-zone active sound profiling is delivered. The fact that the proposed algorithms minimize a number of cost functions associated to each error sensor, instead of minimizing a centralized cost function that associates all the sensors in the array, allows the accomplishment of such a goal.

The possibilities in which the pseudoerror signal can be improved have led to the proposition of a number of multichannel control schemes provided with the coupling compensation mechanism [49, 51, 79]. The main advantage of the so-called multichannel Algorithm 2 relies in its reduced computational demand, which enables it to be implemented in low-power DSP hardware, or even for operation at higher sampling rates. The ability of the frequency-domain version of the multichannel Algorithm 2 relies on controlling the amplitude function of $K / 2-1$ harmonics in a multiharmonic sound, where $K=L$ is the length of the Fourier transform, which equals the order of the adaptive filter per channel. Provided the system matrices defined as $\mathbf{A}=\widehat{\mathbf{S}}(z)^{H} \mathbf{S}(z)$, which are persistently excited at the frequencies of the components of the incoming sound, only shows eigenvalues with positive real parts, the proposed control targets can be attained with no control effort constraints. Otherwise, the stabilization of the algorithm, which has been proposed via control effort constraints, comes at the cost of reducing the performance of the controller, as given in terms of both the attainment of control targets and rejection of cross-channel interferences. Even so, this stabilization strategy does not hinder the operation of the counterbalancing scheme.

Figure 6 illustrates each of the calculations needed for the frequency-domain version of the multichannel Algorithm 2 to complete an iteration. These calculations are also summarized in Table 1, which describes the needed amount of real-valued multiplications and additions needed for accomplishing one iteration.

\section{Experimental results}

This section presents a laboratory experiment, intended to demonstrate the effectiveness of the proposed framework in enhancing the SQ of stationary hybrid electric powertrain noise, for four relevant locations inside a scaled vehicle mock-up. The low computational cost of the selected control algorithm has enabled its on-line implementation at a high sampling rate, close to $6 \mathrm{kHz}$, compatible with that of the realistic stationary hybrid electric powertrain noise.

\subsection{Workbench}

A real 1:3-scaled vehicle mock-up has been instrumented such that the passenger cavity can be excited by structure-borne disturbances. The vehicle mock-up consists of a couple of $3 \mathrm{~mm}$ thick flexible aluminium plates, which emulate the firewall, and a rigid-wall cavity that has been internally conditioned with acoustic-absorbent panels placed on the floor and rear wall, in an attempt to emulate the trimming of a real car. 


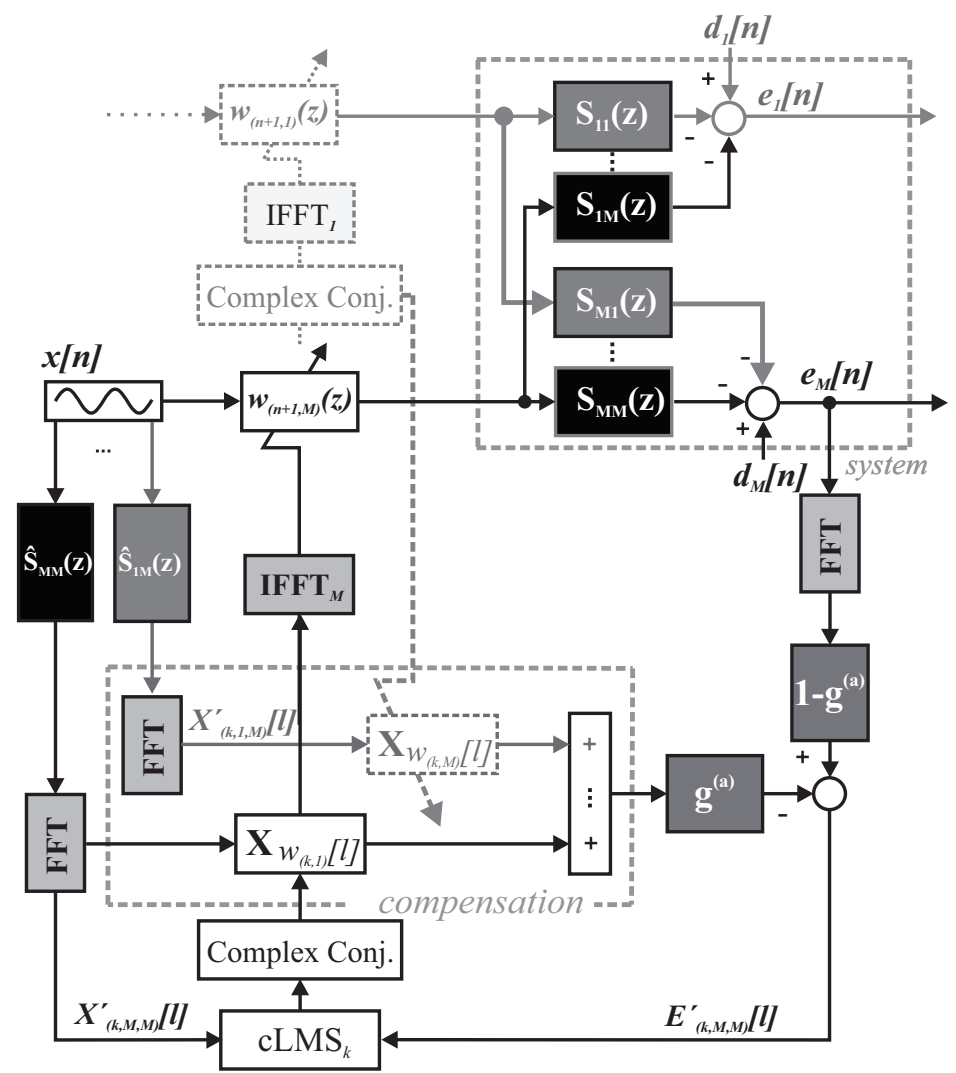

Figure 6: Block diagrams of the multichannel active sound quality control scheme [49].

Table 1: Summary of calculations and computational complexity of the frequency-domain version of the multichannel Algorithm 2 for controlling $L / 2-1$ harmonics, given in real-valued multiplications and additions. The total number of multiplications and additions is given for $M$ channels.

\begin{tabular}{lll}
\hline Equation & Number of Mult. $(\times)$ & Number of Add. (+) \\
\hline$e_{m}[n]=d_{m}[n]-\sum_{j=1}^{M} S_{(m, j)}(z) *\left\{\mathbf{w}_{j}^{T}[n] \mathbf{x}[n]\right\}$ & & - \\
$x_{(m, j=1: M)}^{\prime}[n]=\widehat{\mathbf{S}}_{(m, j=1: M)}[n] \mathbf{x}[n]$ & $M Q$ & $M(Q-1)$ \\
$\mathbf{X}_{(m, j=1: M)}^{\prime}[l]=\mathcal{F}\left\{\left[x_{(m, j=1: M)}^{\prime}[l L-1] \ldots x_{(m, j=1: M)}^{\prime}[l L-L]\right]^{T}\right\}$ & $M L \log _{2} L$ & $M L \log _{2} L$ \\
$\breve{U}_{(k, m)}[l]=\sum_{j=1}^{M} W_{(k, j)}[l] X_{(k, m, j)}^{\prime}[l]$ & $4 M L$ & $2 L(M-1)$ \\
$\mathbf{E}_{m}[l]=\mathcal{F}\left\{\left[e_{m}[l L-1] \ldots e_{m}[l L-L]\right]^{T}\right\}$ & $L \log _{2} L$ & $L \log _{2} L$ \\
$E_{(k, m)}^{\prime}[l]=\left(1-g_{(k, m)}\right) E_{(k, m)}[l]-g_{(k, m)} \breve{U}_{(k, m)}[l]$ & $4 L$ & $4 L$ \\
$W_{(k, m)}[l+1]=\delta_{(k, m)} W_{(k, m)}[l]+\alpha_{(k, m)}\left\{\bar{X}_{(k, m)}^{\prime}[l] E_{(k, m)}^{\prime}[l]\right\}$ & $12 L$ & $2 L$ \\
$\mathbf{W}_{m}[l+1]=\left[\mathbf{W}_{(k=0, \ldots, K / 2 ; m)}^{T}[l+1], \mathbf{W}_{(k=1, \ldots, K / 2-1 ; m)}^{H}[l+1]\right]^{T}$ & - & - \\
$\mathbf{w}_{m}[n+1]=\mathcal{F}-1\left\{\mathbf{W}_{m}[l+1]\right\}$ & $L \log _{2} L$ & $L \log _{2} L$ \\
$u_{m}[n]=\mathbf{w}_{m}^{T}[n] \mathbf{x}[n]$ & $L$ & $L-1$ \\
\hline Total number of multiplications and additions: & $M\left\{M\left[Q+L\left(\log _{2} L+4\right)\right]\right.$ & $M\left\{M\left[Q+L\left(\log _{2} L+2\right)-1\right]\right.$ \\
\hline
\end{tabular}


Figure 7(a) illustrates the exterior of the mock-up, together with the control \& measurement equipment. The cavity has been excited with a PZT patch MIDE QuickPack QP10N. The PZT patch has been installed in the middle of the upper edge of the bigger firewall partition, as close as possible to the clamping mechanism, in order to guarantee a good authority over the main modes. In this way, the sound that reaches the microphones in the passenger compartment is a result of the structural borne excitation of this vibro-acoustic system. The PZT is capable of exciting the cavity at a bandwidth that is compatible with the noise emitted by the hybrid electric powertrain. Figure 7(b) shows the interior of the cavity, which has been instrumented with four microphones placed at locations close to the actual driver and passengers positions. Four loudspeakers have also been placed at locations that emulate the actual positions of the doors of a commercial sedan vehicle. Each microphone has been associated with the closest loudspeaker in the cavity. Figures 7(c) and (d) show schematic views with the main dimensions of the mock-up.

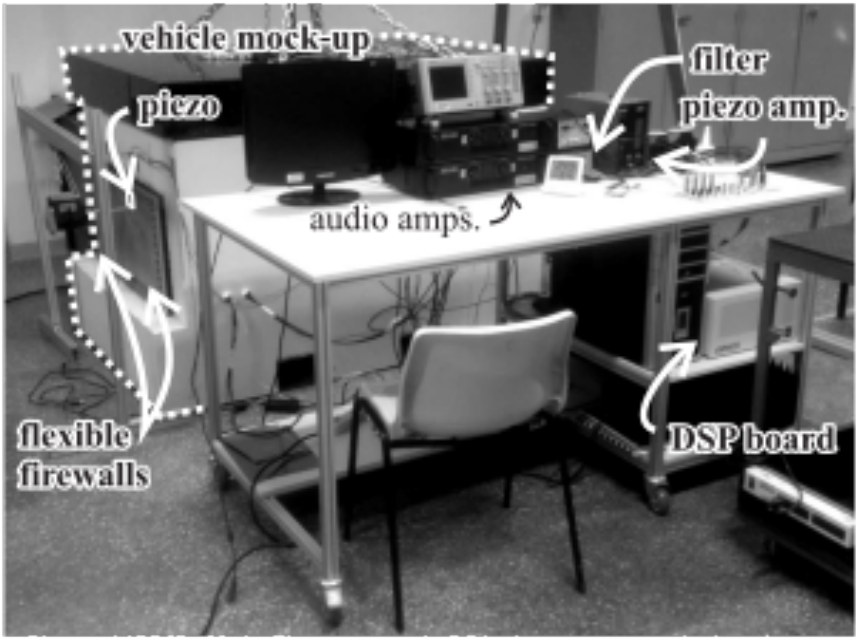

(a)

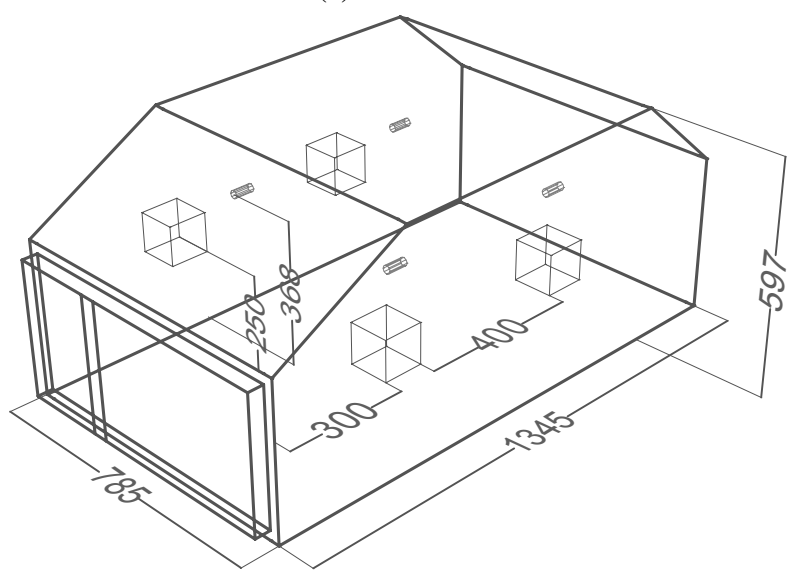

(c)

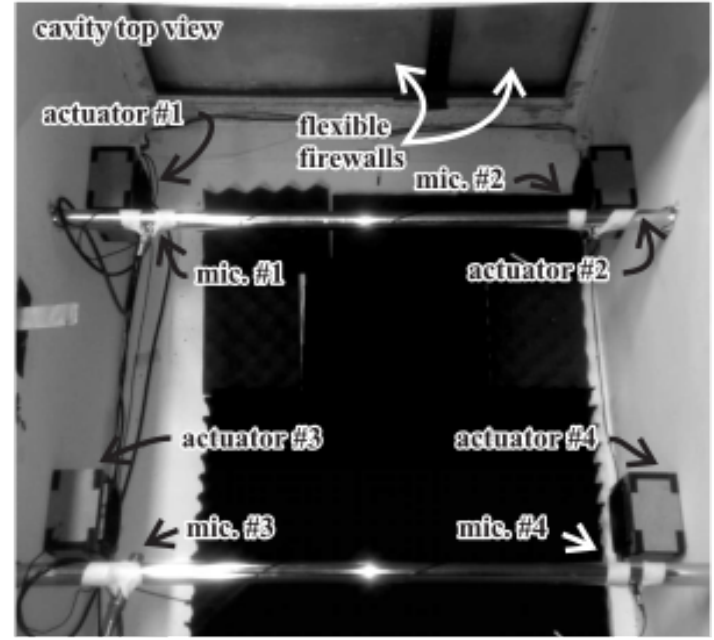

(b)

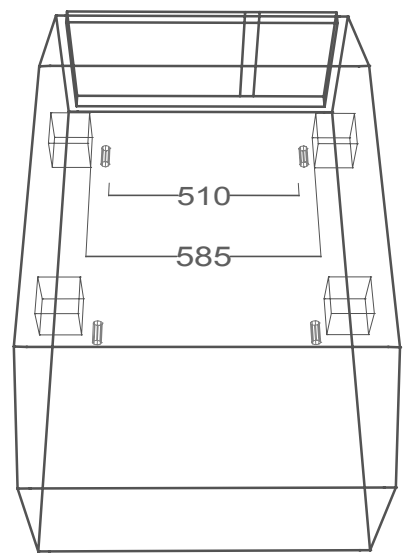

(d)

Figure 7: Images of the experimental workbench: (a) exterior; (b) interior; (c) schematics - southeast isometric view of cavity interior, dimensions given in $\mathrm{mm}$; (d) schematics - top view of cavity interior, dimensions given in $\mathrm{mm}$.

Antialiasing and reconstruction analog bandpass filters have been implemented for each acquisition channel, as follows: the high-pass cutoff frequency is adjusted at $10 \mathrm{~Hz}$, while the low-pass cutoff frequency has been adjusted at $3 \mathrm{kHz}$. The low-pass cutoff frequency is compatible with the sampling rate of the experiment, which, according to the frequencies of the targeted tones in the stationary hybrid electric powertrain noise to be tackled, has been found as 
$5899.38 \mathrm{~Hz}$. This sampling rate has been found by making:

$$
\min (\operatorname{Freq}(i+1)-\operatorname{Freq}(i)) \times L \leqslant \operatorname{Freq}(R),
$$

where $\min ($ Freq $(i+1)-$ Freq $(i))$ is the minimum difference between contiguous tones that is found in the noise; $\operatorname{Freq}(R)$ is the frequency of the highest tone in the noise; and $L=38$ is the length of the discrete Fourier transform that holds the expression for the present experiment. From this relation, the frequencies of the tones determined by the EE-based sensitivity analyses for driving the MOO are also obtained, by making $(F s / L \times\{1,4,5,6,12,18\})$, cf. subsections 4.5.1 and 4.5.2.

\subsection{Implementation of multi-objective routines and multichannel ASQC algorithms}

The scripts for the MOO stage have been written as .m codes for MATLAB. With regard to the MOO solver, the routines provided by the MATLAB's Global Optimization Toolbox [80], equipped with customized genetic operators (SBX crossover and polynomial mutation), have been used for implementing the NSGA-II solver.

The scripts for the control algorithms have been written as C S-functions for Simulink, and compiled for the DSP platform dSPACE 1006. This platform, available at the Dynamics Laboratory from the São Carlos School of Engineering of the University of São Paulo, is equipped with a 16-channel, 16-bit analog-digital converter (ADC), and a 5-channel, 12-bit digital-analog converter (DAC). The control units have been programmed so as to operate in a serial form. In other words, the generation of the control outputs should wait until the communication flow from Channel 1 to Channel 4 had been completed.

\subsection{Dynamics of the acoustic plant}

Since the mock-up is airborne-sealed, plant variations due to changes in the acoustic domain are mostly avoided [81]. Hence, a single measurement of the acoustic control plant was carried out before each experimental run, just to account for long-term environmental changes (air temperature and humidity). The experiments were carried out at times in which the external temperature was likely to remain constant, as well as the background noise was likely to be low. The impulse responses from each actuator to all the microphones within the cavity were identified by following a LMS-based system identification algorithm [82], which uses Gaussian noise as the excitation signal. The length of the impulse responses, $Q$, has been determined in a trial-and-error basis, in an attempt to have an accurate representation of the system dynamics, while keeping the computational cost as low as possible. The frequency response functions (FRFs) are calculated based on the Fourier Transform of the identified FIR filters. The norm-2 condition number is calculated from the matrix $\mathbf{A}(f)=\widehat{\boldsymbol{S}}(f)^{H} \mathbf{S}(f)$, where $\mathbf{S}(f)$ is the matrix arranged at the frequency $f$, by putting the direct paths terms of the FRFs onto the main diagonal, and the crossed terms of the FRFs at the corresponding entries in the matrix. The subsequent calculation of the phase delay function, $\Delta_{j}$, is detailed in Subsection 4.4.

In Figs. 8(a), (b) and (d), which illustrate the magnitude, phase and phase delay of the FRFs, respectively, bold continuous lines represent the direct secondary paths, i.e. transfer paths between a given loudspeaker and the closest microphone placed within the cavity; while dotted lines represent the crossed secondary paths, i.e. transfer paths between a given loudspeaker and other microphones. Moreover, in Fig. 8(c), the bold continuous line represents the norm-2 condition number of the control matrices, as a function of the frequency, while dark dots highlight control matrices at which the operation of the proposed multichannel arrays is predicted to require stabilization.

Generally speaking, as each microphone has been placed as close as possible to its corresponding loudspeaker, magnitude functions with the direct paths larger than the one of the crossed paths have been realized. This feature of the SAP positioning is still more evident in the absolute phase and phase delay functions, where the crossed paths are larger (in absolute value) than the direct ones. However, some magnitude values of the crossed paths that are larger than the ones of the direct paths can still be found. This follows from the observability each microphone may have of the modes being excited by other than its corresponding loudspeaker.

\subsection{Parameters setup for control schemes}

With the dynamics of the acoustic plant properly identified, the step sizes, $\boldsymbol{\mu}$, as well as the control effort constraints, $\boldsymbol{\delta}$, can be estimated for each single control unit in the multichannel algorithm. These parameters are calculated on the premise of obtaining the fastest convergence, while ensuring stable operation. Thus, at the frequencies 


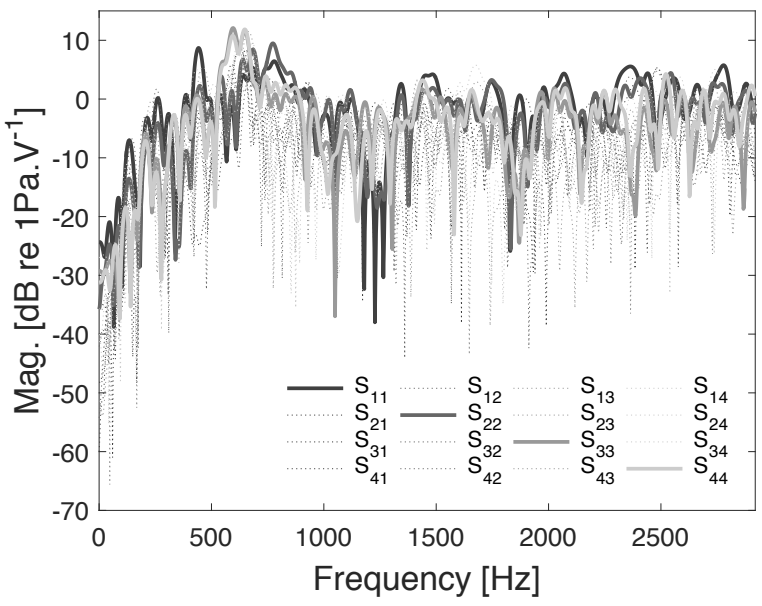

(a)

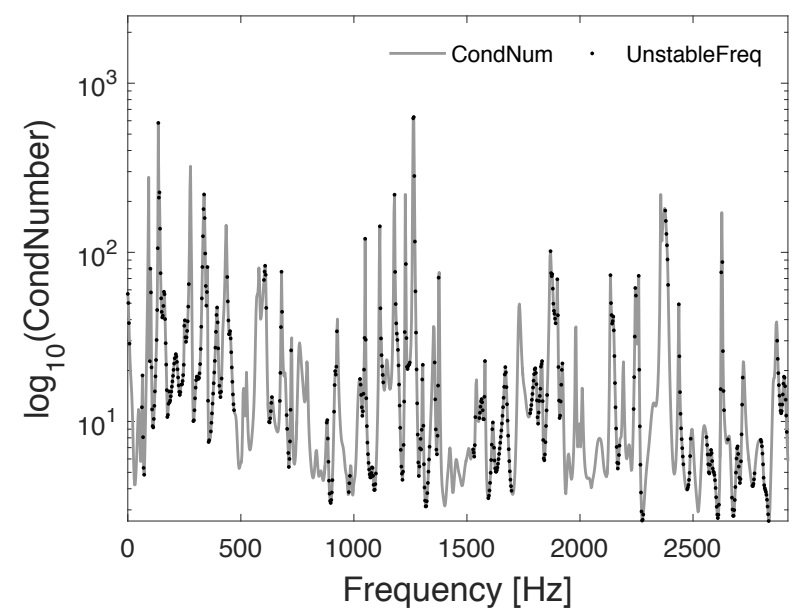

(c)

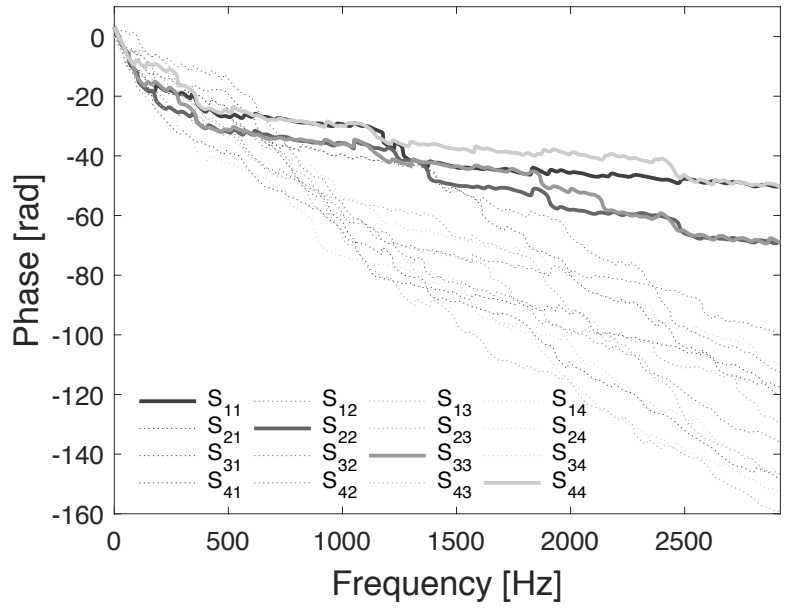

(b)

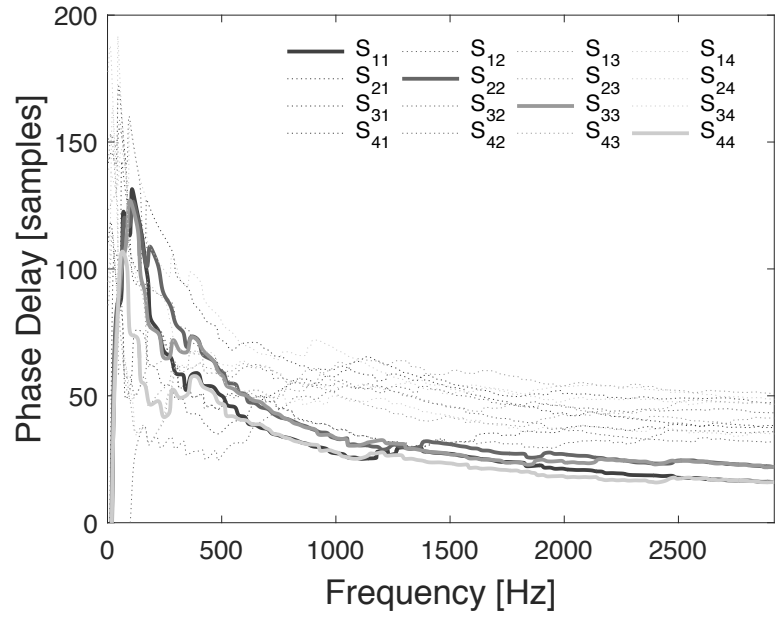

(d)

Figure 8: Frequency response function and related functions of the MIMO control array: (a) magnitude; (b) phase; (c) norm-2 condition number of matrix $\mathbf{A}(z)$; (d) phase delay.

in which the matrix $\mathbf{A}(z)=\widehat{\boldsymbol{S}}(z)^{H} \mathbf{S}(z)$ shows eigenvalues with negative real parts, the regularization parameters for each control unit were calculated by making:

$$
\boldsymbol{\delta}=2 \operatorname{diag}(|\widehat{\mathbf{S}}(z)|) L,
$$

where $L$ is the length of the FIR filter that acts as the adaptive controller. It is worth recalling that, for the frequencydomain control schemes, $L=K$, where $K$ is the length of the discrete Fourier transform.

The step-sizes for the LMS algorithms were calculated by using the following adapted expression [83]:

$$
\mu_{j}=\frac{0.5}{L N B_{j}\left(2 \Delta_{j}+1\right)},
$$

where $\mu$ is given for each $j=1: M$ control unit as a function of the length of the adaptive filter $L$; the number of tones to be controlled $N$; the power $B$ of the reference signal, as "seen" by controller $j$; and the minimum phase delay 
$\Delta$ amongst a given sensor and all actuators. The last two entities, namely $B$ and $\Delta$, are calculated as follows:

$$
B_{j}=\sqrt{\sum_{j=1}^{M}\left|\widehat{\mathbf{S}}_{(m, j)}(z)\right|^{2}} ; \quad \Delta_{j}=\min \left(-\frac{\boldsymbol{\phi}_{(m, j=1: M)}}{\mathbf{\Omega}}\right),
$$

where $\phi_{(m, j=1: M)}$ is the unwrapped absolute phase of the secondary paths, and $\boldsymbol{\Omega}$ is the normalized frequency vector $2 \pi \mathbf{f} / F s$, at which the secondary paths have been identified.

\subsection{Sound quality balancing of the interior hybrid electric powertrain noise for multiple sensor locations}

This section presents the experimental results for the MIMO control scenario. Each of the steps of the proposed framework for approaching the SQ of the realistic hybrid electric powertrain noise are briefly mentioned, in order to sketch an outline of the procedures and main goal of the experiment:

- The first procedure consists on the measurement of the noise at the relevant locations in the cavity. Twenty seconds long measurements have been used. Frequency-domain data of the noise, e.g. amplitude, frequency and relative-phase of each relevant tone in the noise, are extracted at this stage.

- SQ algorithms are used for determining the scores of the noise, for each sensor location.

- Sensitivity analyses based on the EE method, as well as the MOO routines, are independently executed for each sensor location.

- The controller is eventually implemented, by using the optimal amplitude gain factors for each targeted tone, which are expected to drive the sound towards enhanced SQ goals. Therefore, the control target pursued in this Experiment is to implement the optimal solutions at each of the four targeted locations, with the aid of the multichannel cost-effective Algorithm 2, which features compensation for cross-channel interferences.

The procedure for the data acquisition is as follows: $2 \mathrm{~s}$ of latency are used for attaining steady-state conditions, after which the controller is turned on for $8 \mathrm{~s}$. The amplitude gain factors used during control operation are those provided by the MOO stage. Off-line analyses on the norm-2 condition number of the physical array suggested that the controllers will be able to accomplish convergence and steady state in a short timeframe, since the tones of the primary sound requiring control excite well-conditioned, but unstable, control system matrices. It is worth noticing that all the system frequencies requiring control for this experiment needed to be constrained, in order to guarantee stable operation, which in turn had an impact on the control performance.

\subsubsection{Measurement and $S Q$ calculation}

The first step is concerned with the measurement and the SQ calculation of the primary sound, which must be repeated for each targeted location. The primary sound has been synthesized at a sampling rate of $F s=5899.38$ $\mathrm{Hz}$, which is compatible with the number of Fourier bins, $L=38$, of the discrete Fourier transform algorithm into each channel of the multichannel control algorithm, cf. Fig. 6, as well as with the frequencies of the targeted tones in the sound. The Fourier bins at which the targeted frequencies lie into are: $154.98 \mathrm{~Hz} \rightarrow 1,619.94 \mathrm{~Hz} \rightarrow 4,774.92$ $\mathrm{Hz} \rightarrow 5,929.90 \mathrm{~Hz} \rightarrow 6,1862.31 \mathrm{~Hz} \rightarrow 12$ and $2792.21 \mathrm{~Hz} \rightarrow 18$. A list of the targeted system frequencies with additional details can be found in Table 3 .

Some data post-processing steps should be carried out, e.g. order-tracking techniques [84], in order to extract the frequencies of the tones, as well as their amplitudes and relative phases. From this procedure, it has been possible to identify 43 tones, whose amplitude are larger than zero $\mathrm{dB}_{S P L}$. It is important to notice that not all of the tones should fall into each single Fourier bin, but only the ones that should be addressed, as prescribed by the sensitivity analyses. Figure 9 illustrates the magnitude function that is extracted from the measurements of the primary sound, at each of the four targeted locations in the cavity.

The SQ scores of the original primary sound are calculated by following the time-domain models, cf. Figs. 1, 2, 3 and 4, and they are reported in Table 2. The Sharpness metric has been calculated with the Zwicker's weights, and the Tonality metric with the Shin et al. [41] weighting formula. As soon as the time-domain SQ metrics are assessed, the frequency-domain algorithms are calibrated. These calibrated algorithms are to be used in the following MOO procedures. 


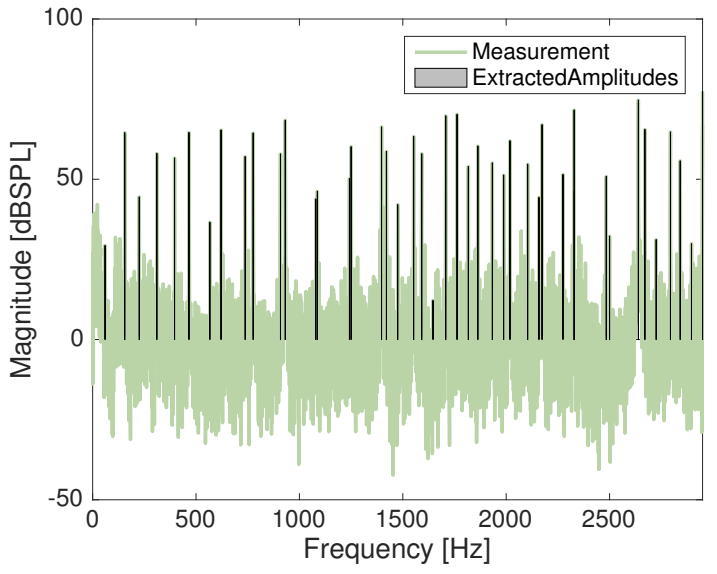

(a)

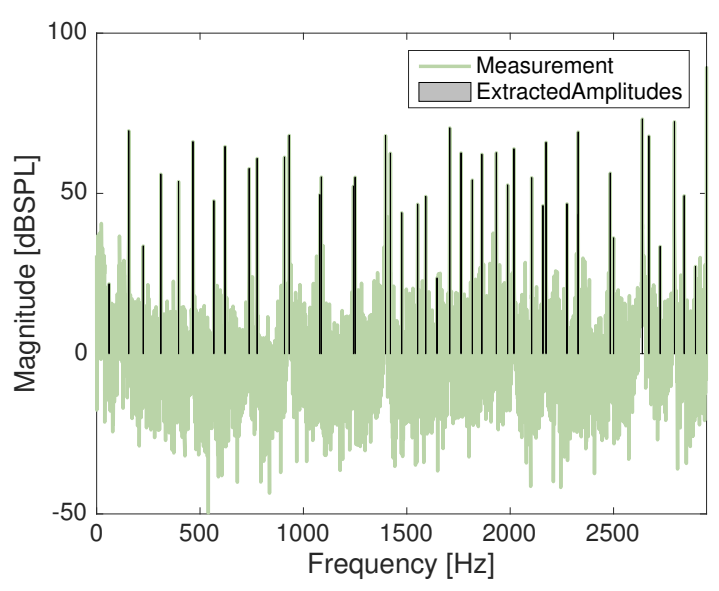

(c)

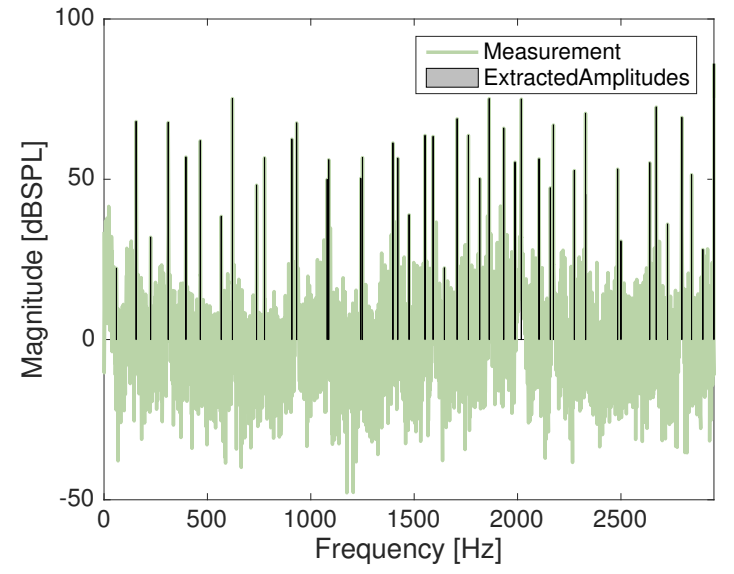

(b)

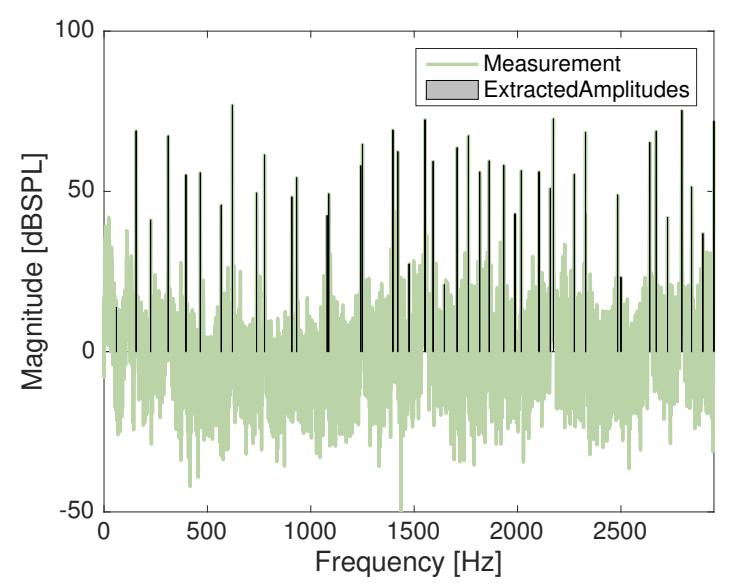

(d)

Figure 9: Magnitude functions of the measured primary sound at: (a) Ch. 1; (b) Ch. 2; (c) Ch. 3; (d) Ch. 4.

Table 2: SQ scores of the primary sound, as determined at each location.

\begin{tabular}{ccccc}
\hline Position & Loudness [sone] & Roughness [asper] & Sharpness [acum] & Tonality [SP] \\
\hline $\mathbf{1}$ & 38.88 & 0.66 & 1.29 & 0.96 \\
$\mathbf{2}$ & 50.00 & 0.68 & 1.42 & 0.90 \\
$\mathbf{3}$ & 49.60 & 0.64 & 1.59 & 0.97 \\
$\mathbf{4}$ & 42.73 & 0.79 & 1.21 & 0.96 \\
\hline
\end{tabular}

\subsubsection{Multi-objective $S Q$ optimization}

The composition of the decision space has been carried out by means of the sensitivity analyses presented in Section 2. Figure 10 illustrates the results of the EE-based sensitivity analysis, discriminated by sensor locations. On the basis of the most involved tones in causing large output variations on each single SQ metric, it has been possible to compose a set of decision variables for each position, such that the amount of tones to be controlled is way smaller than the total amount of tones in the sound.

Now that the decision space has been composed for each single position, the MOO stage takes place in an in- 

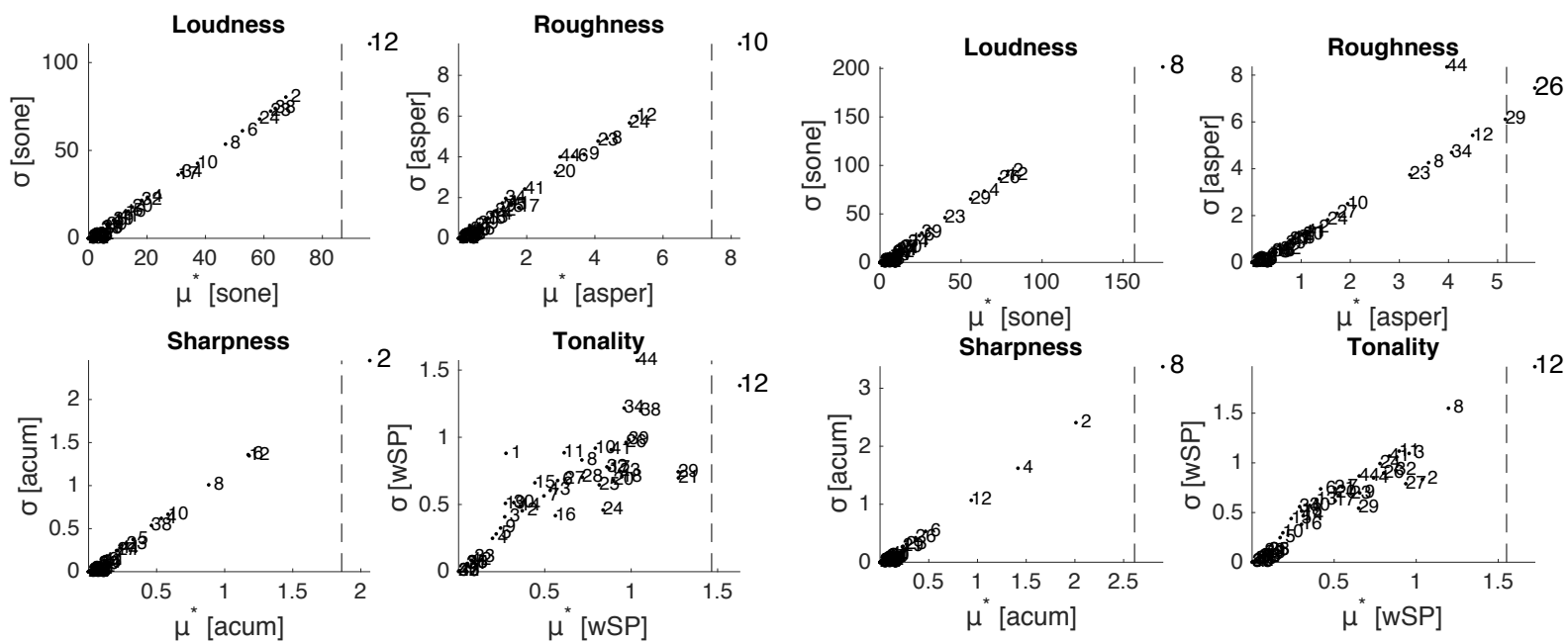

(a)

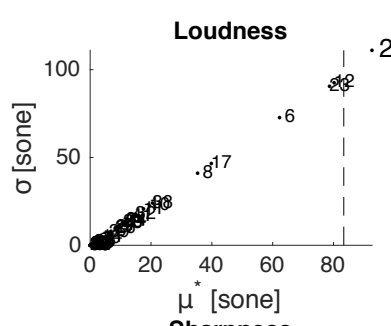

(a)

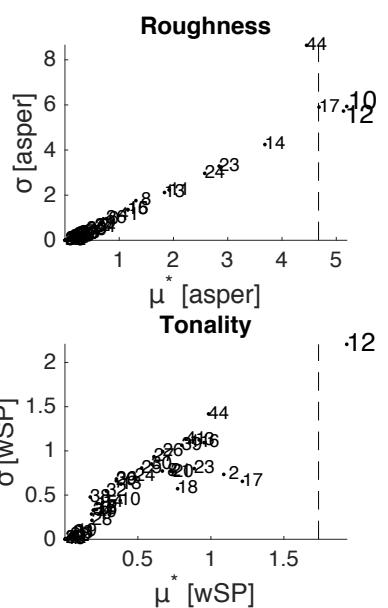

(c) (b)
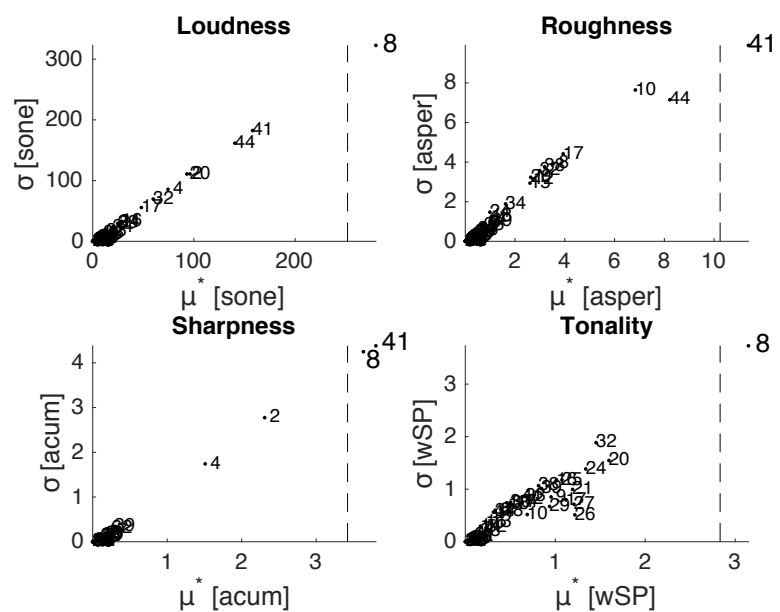

(d)

Figure 10: Sensitivity analyses of the SQ metrics, based on the method of the Elementary Effects, for each position: (a) Ch. 1; (b) Ch. 2; (c) Ch. 3; (d) Ch. 4.

dependent fashion. The results considered for implementation were the ones of the NSGA-II algorithm. Figures 11 and 12 illustrates the scatter-plot matrices obtained after concurrently minimizing and maximizing the associated SQ vector, at each location. In the Figures, the big red dot refers to the original SQ scores of the measured primary sound. The feasible searching space, which has been brought up by extensive search procedures applied on the SQ vector, is represented by using little light-gray dots. The results of the MOO have been discriminated by using black dots for illustrating the concurrent minimization, and light-blue dots for plotting the concurrent maximization.

In general terms, the Pareto fronts obtained for the four locations of interest seem not to cover evenly both the lower and upper edges of the feasible space. Even so, the solutions selected for implementation, which have been highlighted by using color markers in Figs. 11 and 12, are indeed located at the extrema of the optimal Pareto fronts, which also match the ones of the feasible space. It is important to notice that the choice of the extreme values ensures that the maximum and the minimum feasible values of each SQ metric will be tested, while keeping the scores of the remaining metrics still at optimal levels. These solutions at the extrema of the feasible space are the ones that drive the primary sound to substantial changes of its SQ, so they might become relevant in automotive applications.

From the Pareto fronts in Figs. 11 and 12, ten optimal SQ scores have been extracted for implementation, five of 

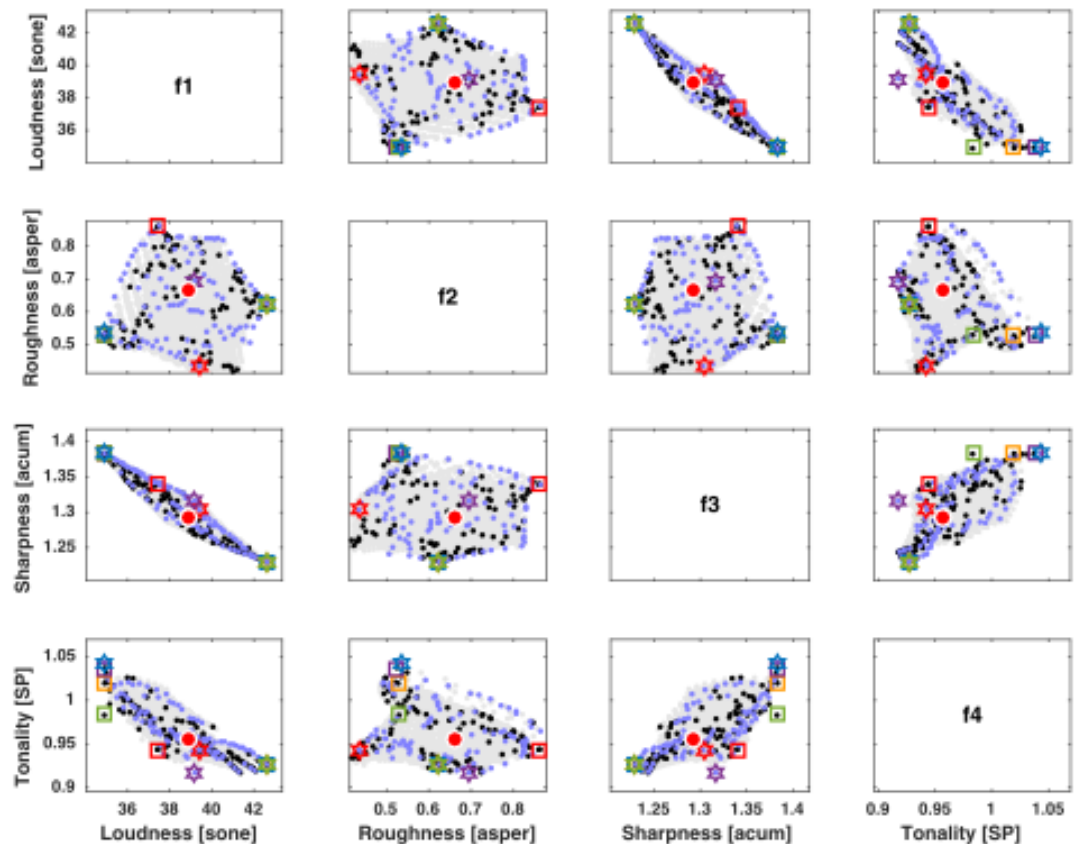

(a)
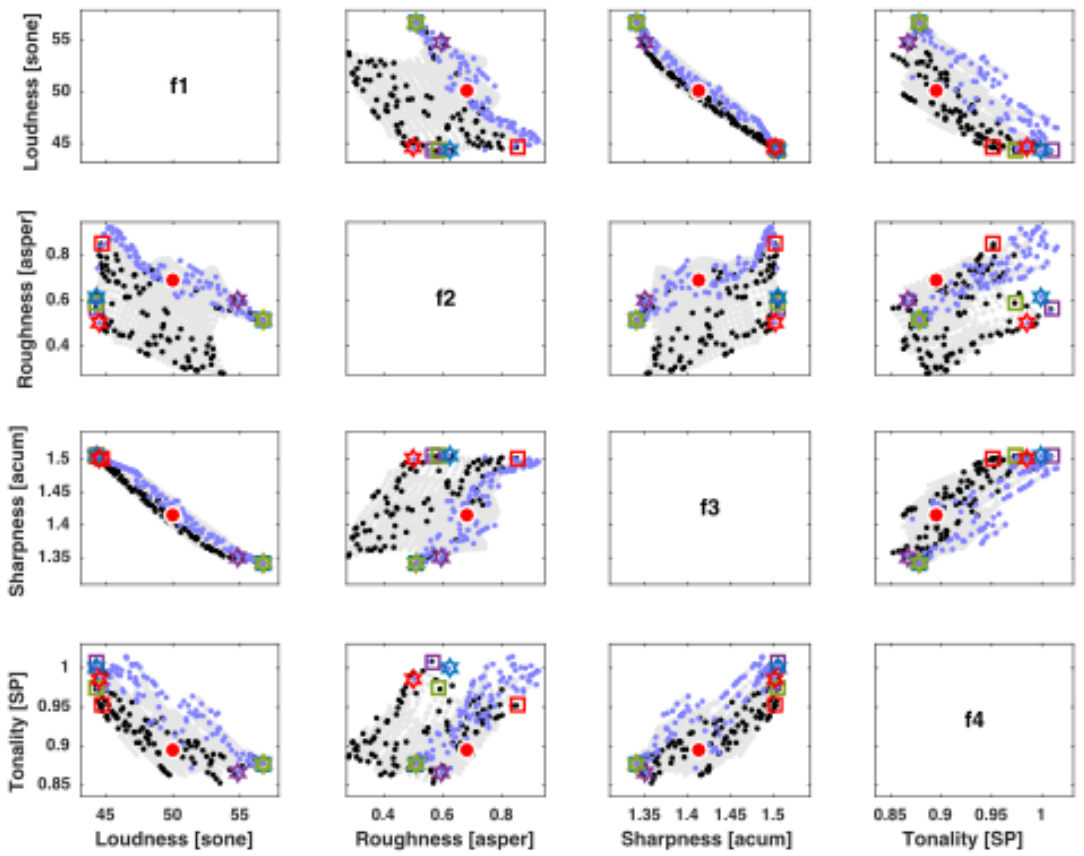

(b)

Figure 11: Multi-objective SQ optimization results: (a) Ch. 1; (b) Ch. 2. Legend: (1) light-gray dots: exhaustive search; (2) black dots: minimization; (3) light-blue dots: maximization; (4) red dot: original SQ scores; (5 9) optimum/attained pairs (color squares): min1: blue; min2: red; min3: orange; min4: purple; min5: green; (10 15) optimum/attained pairs (color stars): max1: blue; max2: red; max3: orange; max4: purple; max5: green.

which belong to the front obtained from the concurrent minimization and the remaining five from the one from the concurrent maximization. This procedure has been carried out for the four locations considered. 

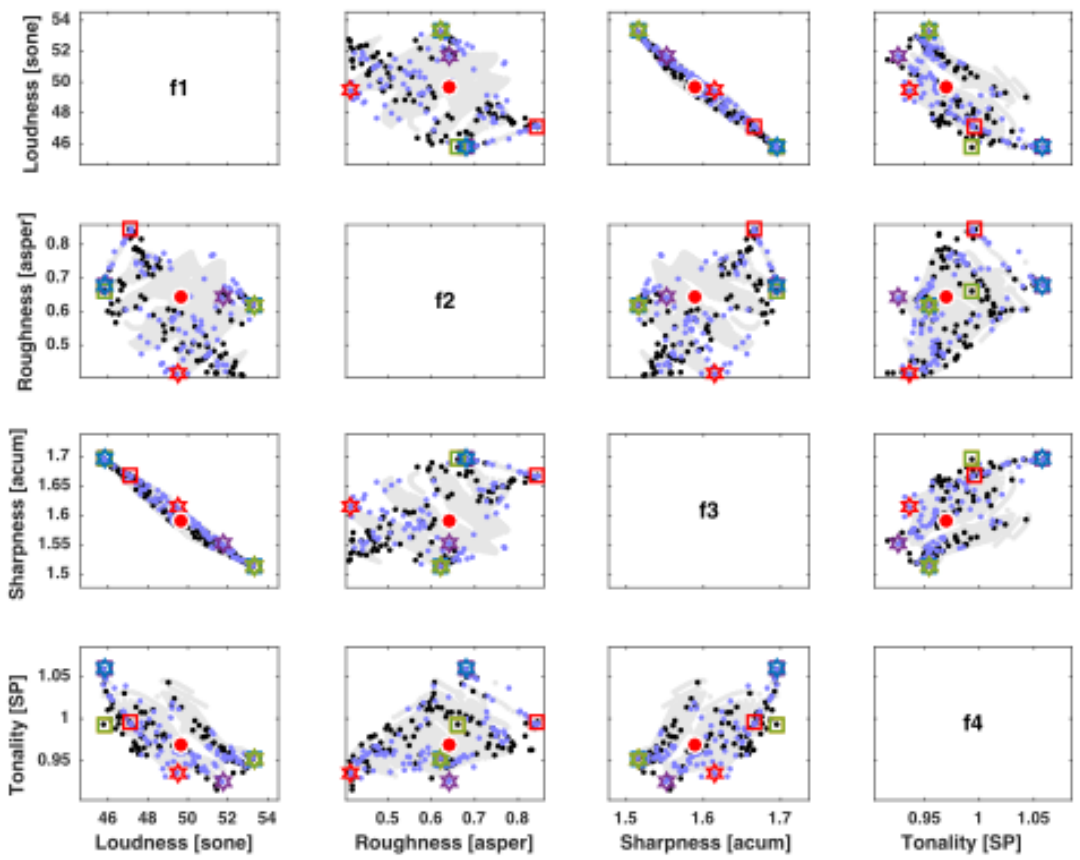

(a)
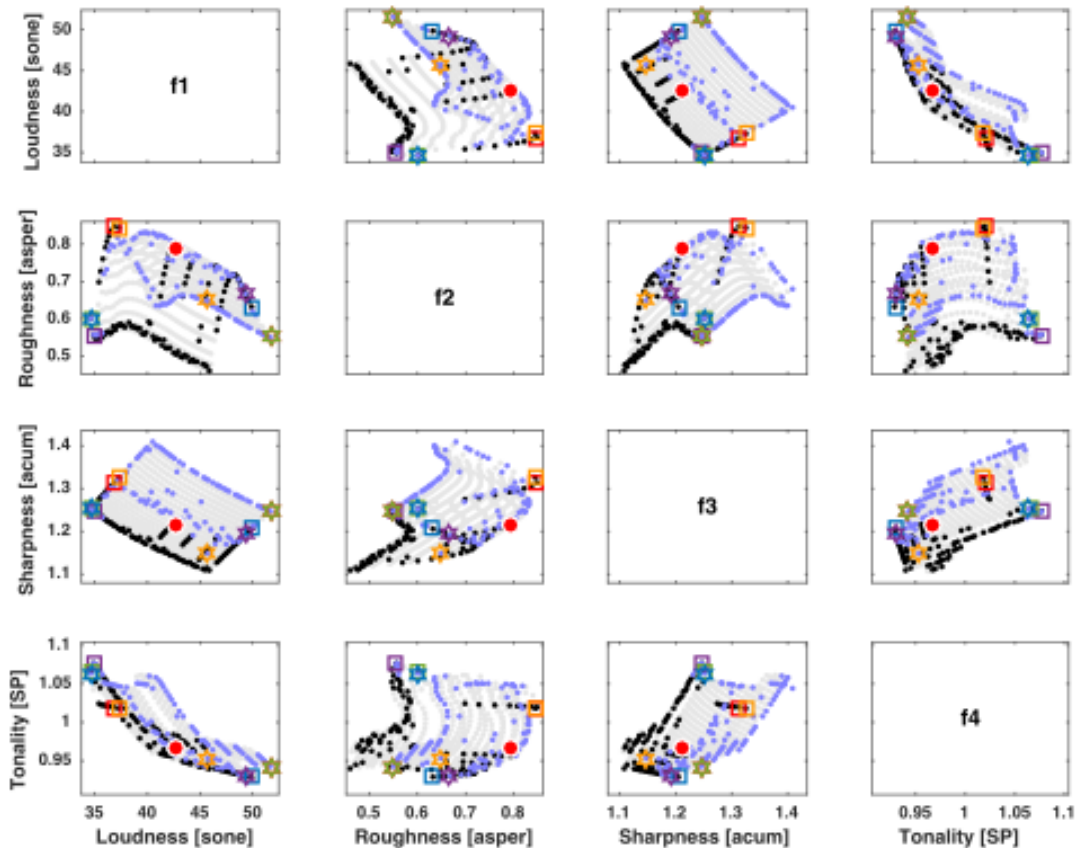

(b)

Figure 12: [con't] Multi-objective SQ optimization results: (a) Ch. 3; (b) Ch. 4. Legend: (1) light-gray dots: exhaustive search; (2) black dots: minimization; (3) light-blue dots: maximization; (4) red dot: original SQ scores; (5 9) optimum/attained pairs (color squares): $\min 1: \mathrm{blue}$; $\min 2$ : red; min3: orange; min4: purple; min5: green; (10 15) optimum/attained pairs (color stars): max1: blue; $\max 2$ : red; $\max 3$ : orange; max4: purple; max5: green. 


\subsubsection{Multichannel ASQC}

With the optimal amplitude gain factors for each tone in the decision sets, as prescribed by the MOO for each targeted location, the next procedure deals with the implementation of the optimal SQ scores. Stability predictions and the calculation of the control parameters were performed with the initial measurement. Table 3 reports on the parameter values for the frequency-domain controller, together with the norm-2 condition number of the system matrices being excited, as well as the smallest real part of the eigenvalues of the matrix $\mathbf{A}(z)=\widehat{\mathbf{S}}(z){ }^{H} \mathbf{S}(z)$. The off-line analyses on the stability of the array predicted that just one tone would require control output limitation, namely, the $154.98 \mathrm{~Hz}$ component. However, the actual control practice verified that all the tones required some level of output constraint in order to guarantee stability, for all the SAPs. Although the practiced system identification was sufficiently accurate for providing good estimates of the step sizes, as well as for ensuring stable, on-line operation of the multichannel controller, it seems that the system representation was not accurate enough to guarantee suitable estimates of the constraints, and hence they were set up in a trial-and-error basis. Since the norm- 2 condition numbers determined for the matrices are small, as compared to the ones obtained in previous experiments, it was expected that the controller would converge quickly, so the allowed timeframe for each observation was limited up to $10 \mathrm{~s}$.

Table 3: The smallest real part of any of the eigenvalues and norm-2 condition number of matrix $\mathbf{A}=\operatorname{diag}(\widehat{\mathbf{S}}(z))^{H} \mathbf{S}(z)$, together with parameters for the LMS adaptive algorithm, at each controlled discrete frequency, for each sensor location.

\begin{tabular}{|c|c|c|c|c|c|c|c|c|c|c|}
\hline \multirow{2}{*}{ Freq. [Hz] } & \multirow{2}{*}{$\operatorname{Re}\left(\lambda_{(\mathbf{A}(z))}\right)$} & \multirow{2}{*}{$\mathrm{CN}$} & \multicolumn{2}{|c|}{ Position 1} & \multicolumn{2}{|c|}{ Position 2} & \multicolumn{2}{|c|}{ Position 3} & \multicolumn{2}{|c|}{ Position 4} \\
\hline & & & $\delta$ & $\mu$ & $\delta$ & $\mu$ & $\delta$ & $\mu$ & $\delta$ & $\mu$ \\
\hline 154.98 & -0.01 & 42.23 & 17.77 & $1.19 e^{-04}$ & 9.01 & $1.13 e^{-04}$ & 7.03 & $6.02 e^{-04}$ & 7.84 & $5.95 e^{-04}$ \\
\hline 619.94 & 0.68 & 12.93 & 10 & $3.35 e^{-06}$ & 10 & $3.54 e^{-06}$ & 10 & $1.37 e^{-06}$ & 10 & $1.33 e^{-06}$ \\
\hline 774.92 & 0.66 & 16.40 & 20 & $4.87 e^{-06}$ & 20 & $2.79 e^{-06}$ & 20 & $1.71 e^{-05}$ & 20 & $1.32 e^{-05}$ \\
\hline 929.90 & 0.23 & 25.94 & 10 & $8.36 e^{-06}$ & 10 & $7.60 e^{-06}$ & 10 & $3.76 e^{-05}$ & 10 & $4.51 e^{-05}$ \\
\hline 1862.31 & 0.04 & 36.86 & 10 & $3.47 e^{-05}$ & 10 & $5.05 e^{-05}$ & 10 & $1.67 e^{-04}$ & 10 & $1.18 e^{-04}$ \\
\hline 2792.21 & 0.90 & 7.71 & 10 & $1.76 e^{-05}$ & 10 & $2.33 e^{-05}$ & 10 & $4.66 e^{-05}$ & 10 & $4.15 e^{-05}$ \\
\hline
\end{tabular}

Figure 13 shows bar plots of the original and final amplitude values after control, wherein each color corresponds to a different solution selected from the MOO. While Fig. 13(a) illustrates the final amplitude values after applying the solutions from the concurrent minimization, Fig. 13(b) illustrates the final amplitude values after applying the solutions from the concurrent maximization. The tones that required cancellation, reduction or amplification, are highlighted by using numbers. The tones that were not highlighted at a certain location are the ones that were supposed to be preserved against cross-channel interferences, as they were not targeted for control by the MOO stage at such given location. While the tones that required modification are clearly profiled, the ones expected to keep their original amplitude values are indeed close to the non-controlled -original- level.

The quantification of the effectiveness of the coupling compensation mechanism at each control location, which is based on the average relative error between the original and final amplitude values after control, yields the following figures: $92 \%$ at Positions 1 and 2, 89\% at Position 3 and $83 \%$ at Position 4. This, in turn, reads an overall efficiency rate of $89 \%$, which means that the average difference between a given amplitude value to be preserved and the final value from applying any of the solutions, at any of the targeted locations, is of $11 \%$. Considering the complexity of the proposed task, of independently equalizing multiple tones simultaneously at different locations, together with the number of possibles sources of uncertainty and intrinsic variability in vibro-acoustic systems, this is considered a quite successful result.

While this percentage value relates to the bare amplitude analysis of the tonal components, the following section deals with the actual impact of those differences on the SQ metrics, which are the actual measure of success in this context. 
Ch. 1

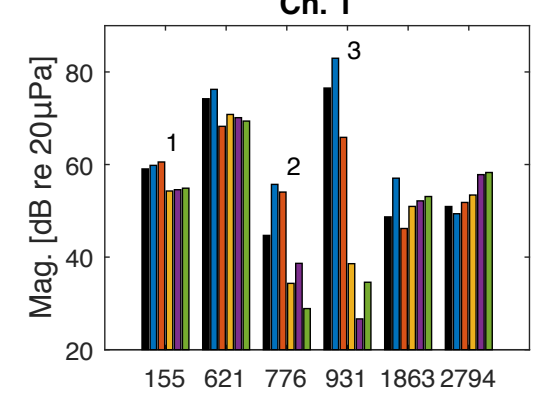

Ch. 3

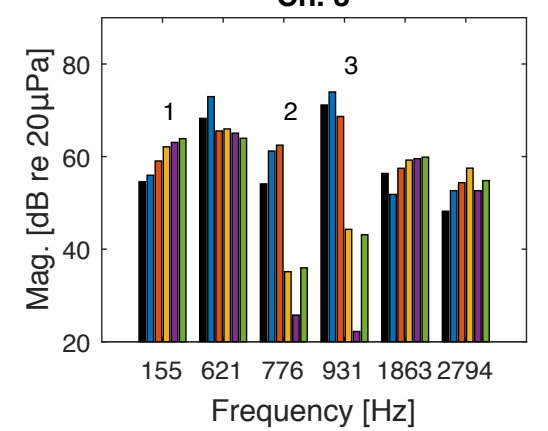

Frequency $[\mathrm{Hz}]$

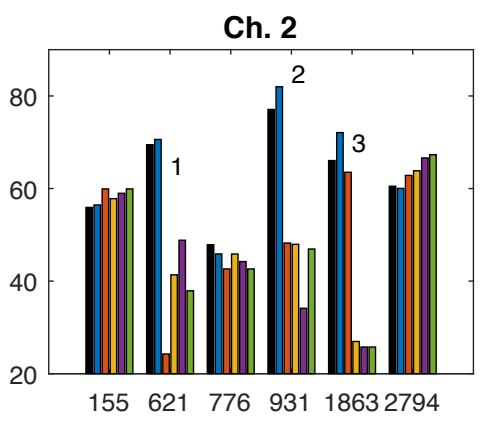

Ch. 4

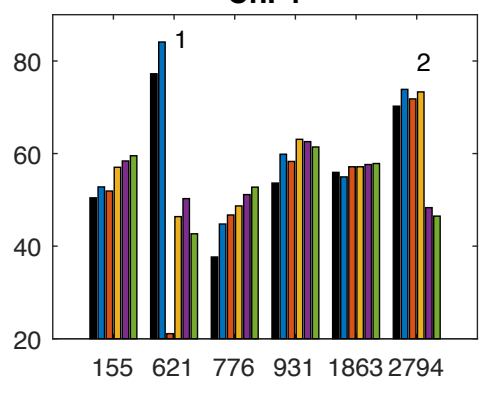

Frequency $[\mathrm{Hz}]$

S2

S3

S4 $\square$ S5

(a)

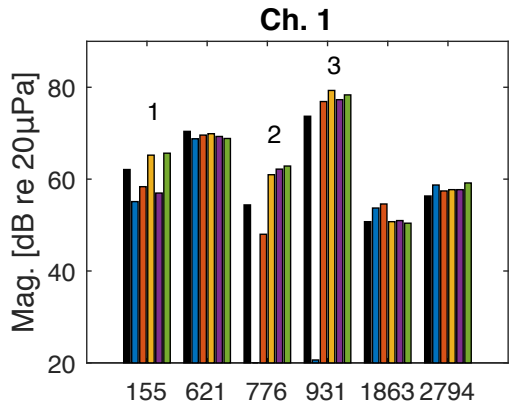

Ch. 2

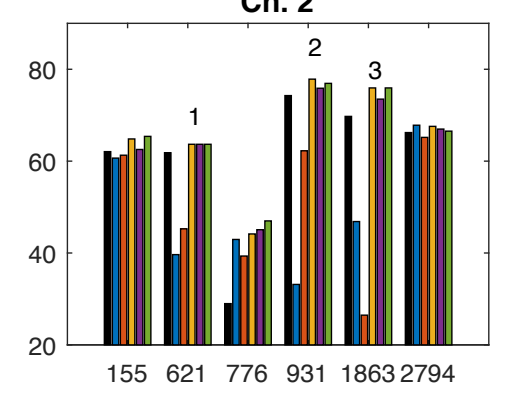

Ch. 4
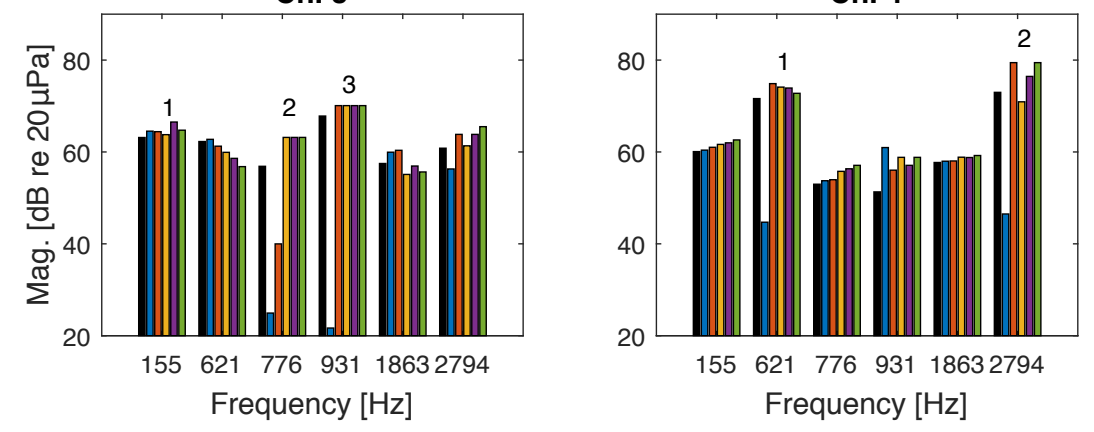

s2

S3

S4 S5

(b)

Figure 13: Grouped bar plot of the final amplitude values, after implementing the optimized SQ targets: (a) solutions from minimization; (b) solutions from maximization. In legends, symbols $S$ \# stand for Solution \#. 


\subsection{4. $S Q$ assessment of the controlled primary sound}

The assessment of the time-domain SQ metrics on the controlled sound will show if the optimized amplitude gain factors effectively drove the SQ scores to enhanced values, at all the targeted locations within the cavity.

Figures 14 and 15 illustrate the time-domain SQ histories that result from applying each of the optimal solutions on the primary sound, as measured at each of the four controlled locations. While Fig. 14 shows the time-domain SQ histories obtained from applying solutions from the concurrent minimization, Fig. 15 shows the time-domain SQ histories obtained from the concurrent maximization. The color convention on those plots match that of Fig. 13, for each selected solution; for instance, the solution Min1 (S1) has the effect illustrated with blue continuous lines, on all the four metrics. It is worth recalling that a certain solution, for instance, solution Minl, was simultaneously applied at the four locations, requiring different operation modes for each tone at each location. This follows from the fact that the MOO and related procedures were independently worked out for each location.
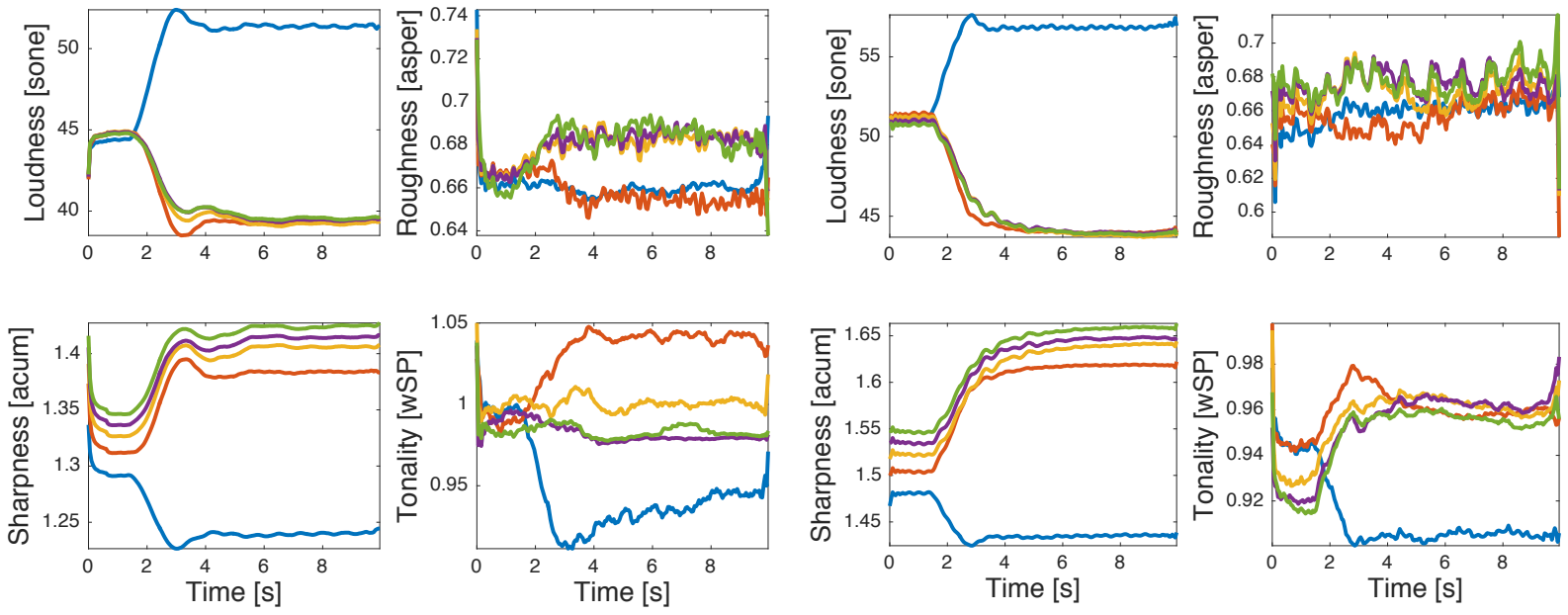

(a)
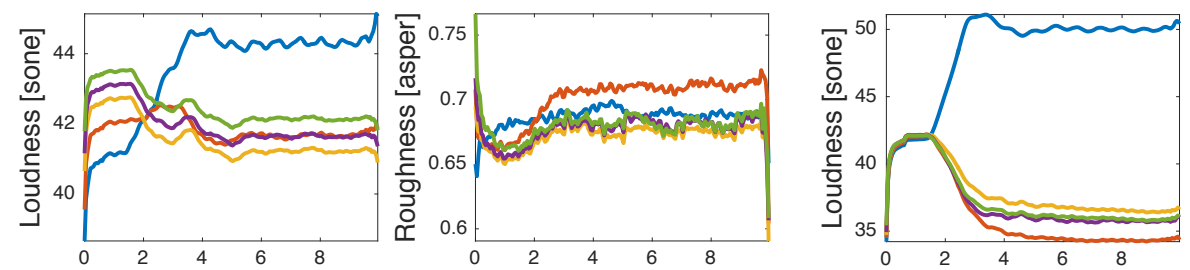

(b)
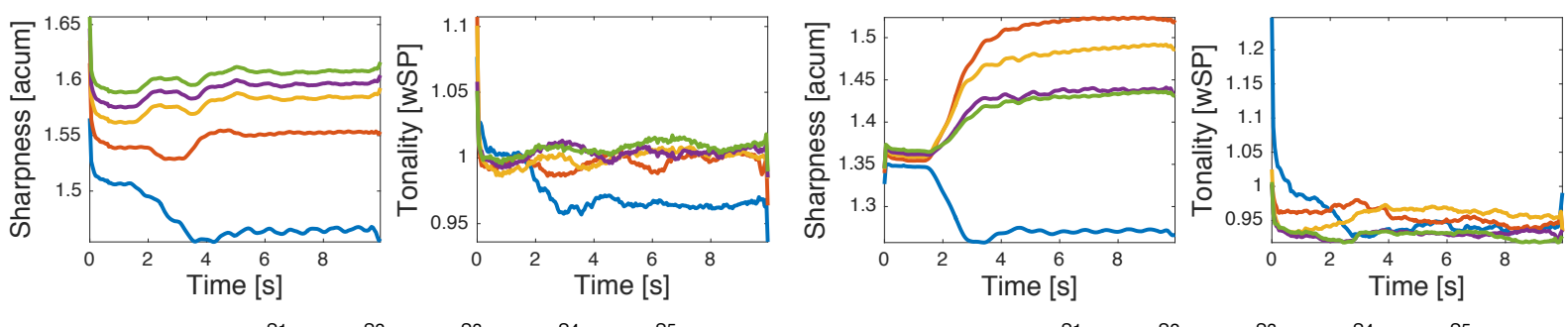

(c)

(d)

Figure 14: Time-domain SQ assessment of the controlled sound, after applying active control guided by concurrent minimization: (a) Ch. 1; (b) Ch. 2; (c) Ch. 3; (d) Ch. 4. In legends, symbols $S \#$ stand for Solution \#.

With regard to the solutions obtained from the concurrent minimization, illustrated in Fig. 14, it is seen that the solution Minl is noticeably different from the remaining ones, resulting in the smallest values for Sharpness and 
Tonality, at the expense of increasing Loudness. Solutions Min2 - Min5 (S2 - S5) also drive the SQ of the primary sound to concurrent optimal scores, always decreasing Loudness. This behavior highlights the intricate relationship and concurrent nature of the SQ metrics. Although significant changes have been achieved for Loudness, Sharpness and Tonality, Roughness remained rather unresponsive to the control actions, in spite of seeming feasible during the MOO stage. With regard to the solutions obtained from the concurrent maximization, illustrated in Fig. 15, it seems that the solution Maxl has noticeably driven Loudness to the maximum value; the remaining solutions also drive the SQ scores to new balances, but their effect seems less drastic. Roughness is again almost unresponsive to any of the solutions implemented on the primary sound, for any of the targeted locations.
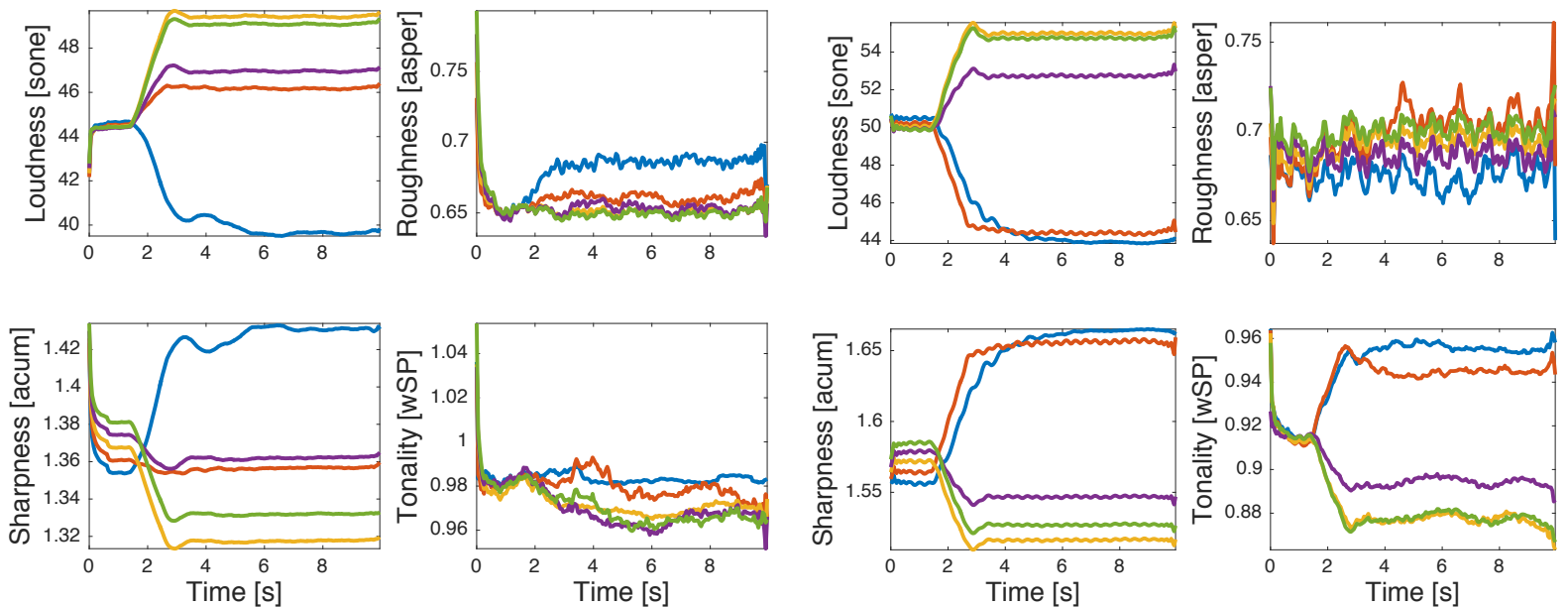

$-\mathrm{s} 1-\mathrm{s} 2$

(a)
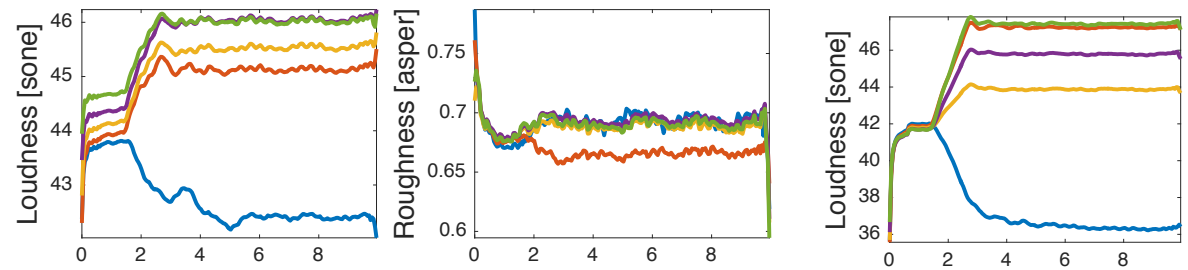

(b)
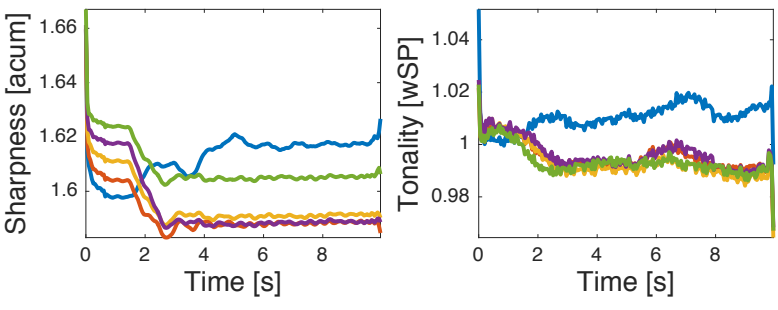

(c)
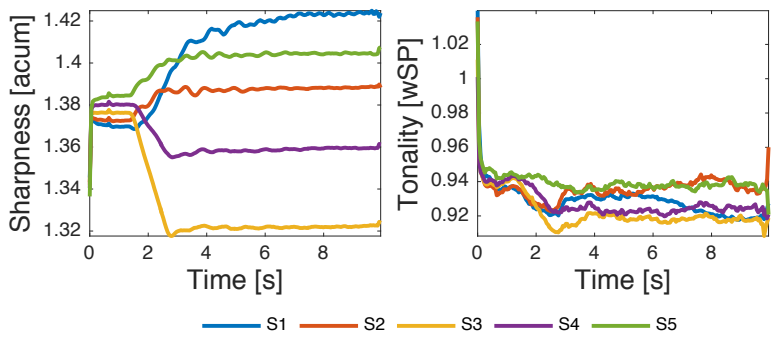

(d)

Figure 15: Time-domain SQ assessment of the controlled sound, after applying active control guided by concurrent maximization: (a) Ch. 1; (b) Ch. 2; (c) Ch. 3; (d) Ch. 4. In legends, symbols $S$ \# stand for Solution \#.

The solutions Min5 and Max5 (S5), which refer to the Global minimum and maximum, respectively, seemed rather difficult to be attained. This is mostly due to the fact that the multichannel algorithm had all its control outputs limited, which hindered the attainment of the most effortful amplitude modes, i.e. cancellation and amplification by twice the original value. 


\subsubsection{Discussion: success percentage and attained solutions in perspective}

Figures 14 and 15 have shown that the SQ scores of the primary sound have been noticeably enhanced at each of the targeted locations, as a function of the optimized amplitude values, which were tackled by the multichannel controller. The results shown so far have also unveiled, though, that the effectiveness of the proposed framework for enhancing the SQ of periodic disturbance was compromised, mainly due to two aspects:

- The strength of the acoustic coupling amongst channels, which in turn enforced the use of control effort constraints which degraded the performance of the multichannel algorithm.

- External influences that caused the sound reaching each SAP to change its time-frequency pattern, from the initial measurement to the active control stages (e.g. significant temperature variations).

At this point, it is convenient to examine the so-called success percentage, which quantifies the extent at which each of the attained SQ scores matched the designed ones. The success percentage allows comparing the scalar values that are provided by the optimized frequency-domain SQ algorithms with the average of the time-domain SQ histories of the controlled disturbance, just after the ASQC scheme attains complete convergence. For instance, in Figs. 14 and 15, it is possible to observe that the multichannel ASQC scheme has attained convergence before $t=5 \mathrm{~s}$. Then, it seems reasonable to take the mean value of the time-domain SQ history for each metric, after the controlled disturbance to have reached the steady state, i.e. to take the average of the time histories from $t=5$ to $t=10 \mathrm{~s}$, in order to establish a comparison with the SQ score provided by the corresponding optimized frequency-domain SQ model. Finally, the success percentage, which is nothing but the relative error between the optimized and the attained SQ score, can be quantified as follows:

$$
\%=\frac{\left(1 / N \sum_{j=1}^{N} F_{T D}(j)\right)-F_{F D}}{F_{F D}} \times 100
$$

where $F_{T D}$ and $F_{F D}$ stand for a given SQ metric that is calculated by means of either time- or frequency-domain algorithms, respectively; and $N$ is the total amount of samples in the selected timeframe of the time-domain SQ history of the metric $F_{T D}$.

After calculating the success percentage between the optimized and attained SQ scores for each single optimized solution, the overall success percentage in attaining any of the designed solutions reads: $89 \%$ for Position $1,90 \%$ for Position 2, 92\% for Position 3 and $91 \%$ for Position 4. This, in turn, yields a total success percentage of $91 \%$, for any of the targeted locations within the cavity. Moreover, it has been determined that the success percentage in attaining Roughness targets is the lowest one, just amounting to $82 \%$, whereas the success percentage of Loudness, Sharpness and Tonality targets are all above $92 \%$.

The impact on the SQ scores of the original sound can also be seen by means of radar plots, shown in Fig. 16 and Fig. 17. For the sake of observation, the axes of the radar plots have been adjusted but kept the same for all the plots within this Figure. These plots allow a direct assessment of a variety of sound quality balances that can be attained on the same primary sound, as dictated by each implemented solution. The most interesting results are those given by the so-called Global minima and maxima, cf. green plots in the cited Figures, which represent the best balance of the SQ metrics. Moreover, as previously highlighted, the solutions related to the Loudness sensation are the ones that have produced the most noticeable changes in the perception of the sound. This observation holds for both concurrent minimization (Fig. 16) and concurrent maximization (Fig. 17).

In Fig. 18, four different results have been extracted from the radar plots in Figs. 16 and 17, in order to highlight some extreme conditions. Figure 18(a) compares the original sound at $\mathrm{Ch}$. 4 with the maximum achieved Loudness (with reduced Roughness and Tonality). Figures 18(b) and (c) compare the maximum Sharpness with the Maximum Loudness scenarios for Channels 1 and 4, respectively. Those two metrics work concurrently in both cases, while Roughness is kept unchanged and Tonality is also increased in Channel 4. Another rather drastic comparison rises from the original sound field and the global minimum achieved at Channel 4, depicted in Fig. 18(d). This solution shows reduced Loudness, Roughness and Tonality, at the expense of increasing Sharpness. As the diamond representing the scores becomes more regular, it is said that the SQ scores of the sound become balanced. While the diamond that represents the original SQ scores is somewhat distorted, showing excess Roughness and Tonality, the obtained green diamond is more regularly composed. Similar results can be observed for the remaining channels after the 
(a)

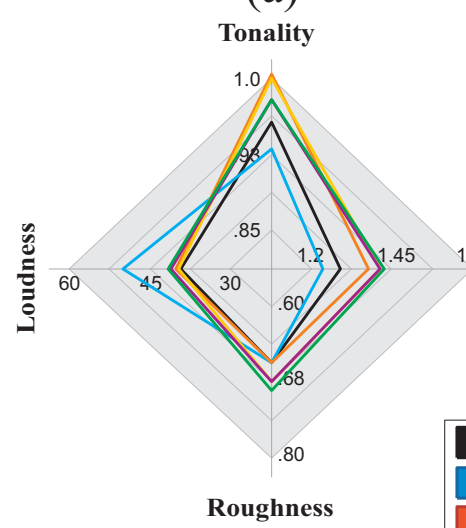

(c)

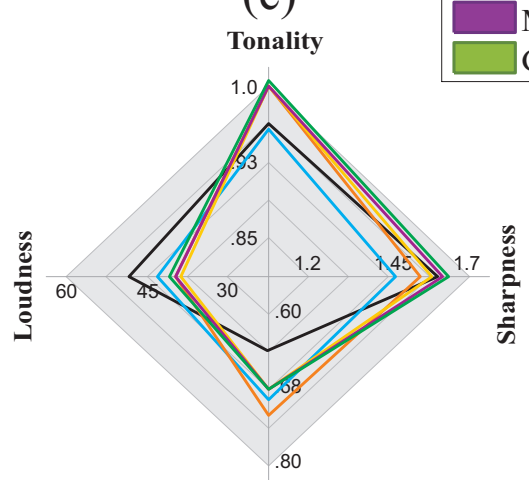

Roughness (b)

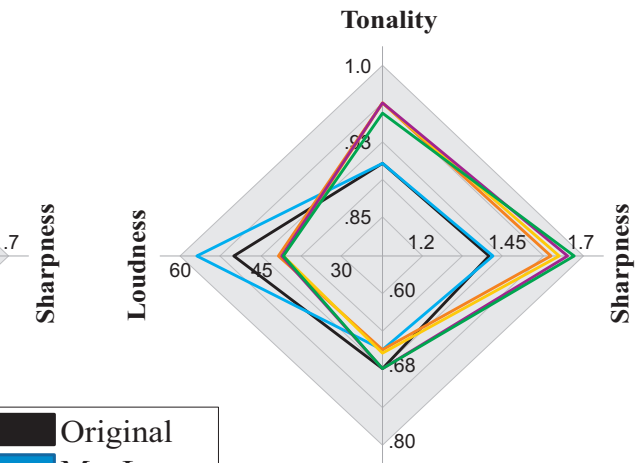

Roughness

(d)

Tonality

MaxT
GlobalMin

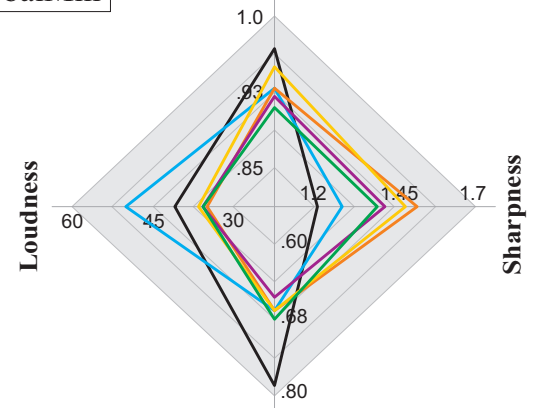

Roughness

Figure 16: Radar plots of the SQ of the controlled primary sound, after applying solutions from the concurrent minimization: (a) Ch. 1; (b) Ch. 2; (c) Ch. 3; (d) Ch. 4.

implementation of the Global Minimum solution, cf. green diamonds in Fig. 16, as well as after the implementation of the Global Maximum solution, cf. green diamonds in Fig. 17.

These results again prove that the SQ scores can be substantially changed for each targeted listener with the proposed technique, even in the presence of strong acoustic coupling, variations in the vibro-acoustic system and control output constraints. The average success percentage of $91 \%$ in experimentally attaining any of the proposed solutions, for all the targeted locations, consistently verifies the effectiveness of the proposed method.

\section{Conclusion}

On the basis that active control techniques are the most versatile ones in profiling periodic sounds, this paper has relied on the extensive use of the class of the active noise controllers referred to as active noise equalizers (ANEs), to propose a framework for enhancing the SQ of the powertrain noise within the passenger cabin in HEVs. The key feature of ANE systems in reshaping the magnitude function of periodic disturbances is extensively used, by means of providing other than pure-reduction solutions, such as equalization, amplification and even preservation of (parts of the) original time-frequency pattern of the noise. The effect of such control actions has been associated to modifications on SQ metrics, such as Loudness, Roughness, Sharpness and Tonality. To this regard, a mathematical expression based on multi-objective optimization (MOO) theory is posed, which associates the aforementioned metrics to the noises of concern. The concurrent optimization of the metrics is seen as a powerful means for guiding the ANE actions, which are expected to drive the SQ of the original soundscape to a variety of enhanced scores. The proposed 
(a)

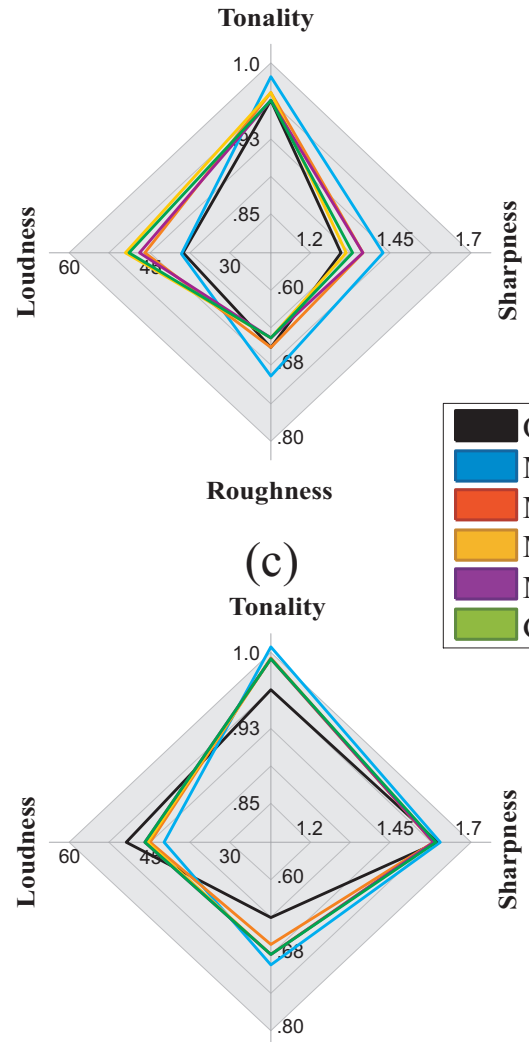

Roughness (b)

Tonality

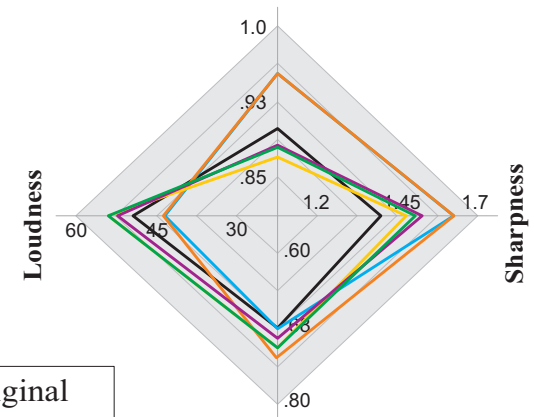

Roughness

(d)

Tonality

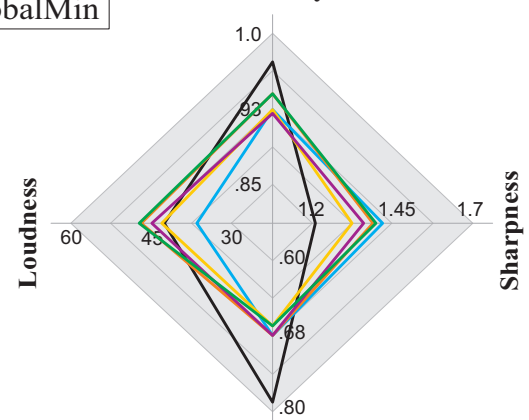

Roughness

Figure 17: Radar plots of the SQ of the controlled primary sound, after applying solutions from the concurrent maximization: (a) Ch. 1; (b) Ch. 2; (c) Ch. 3; (d) Ch. 4.

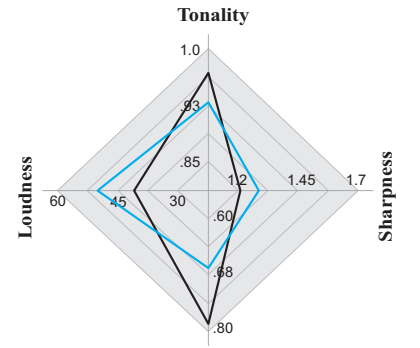

Roughness

(a)

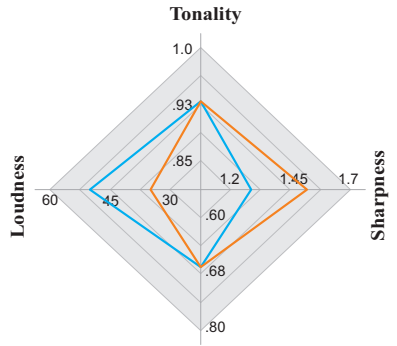

Roughness

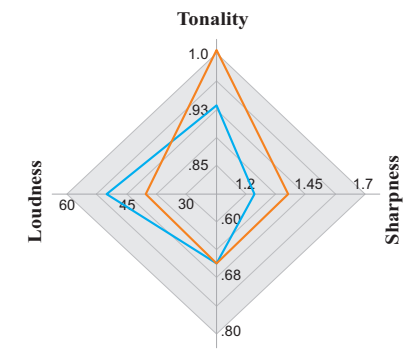

Roughness

(c)

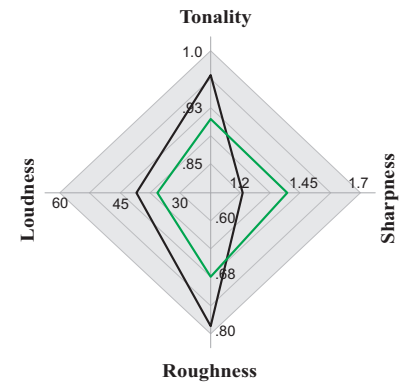

(d)

Figure 18: Comparison between pairs of selected SQ scores: (a) Original/MaxL at Ch. 4; (b) MaxL/MaxR at Ch. 1; (c) MaxL/MaxR at Ch. 4; (d) Original/Global Minimum at Ch. 4.

methodology provides optimal amplitude gain factors, which are implemented via innovative single and multichannel active sound profiling algorithms that deal with cross-channel interferences.

Experiments carried out in a laboratory vehicle mock-up have unveiled that, while Loudness, Sharpness and Tonality targets can be attained with a good level of accuracy, with relative errors between optimal and attained values of $7 \%$, Roughness goals seem to deviate some $20 \%$ from the expected values. These deviations are presumably due to disparities between the time- and frequency-domain algorithms. Even so, since the overall success rate is above $90 \%$, 
the present research concludes that the SQ of the hybrid electric powertrain noise, perceived at multiple locations inside the passenger cavity, can be substantially enhanced, by following the proposed framework.

Future directions are proposed on searching for alternative solutions for the constraint outputs, which would not affect control performance as much, e.g. physical modifications to the SAP; modifications to the control algorithm in order to include (part) of the coupling terms. An extension of the present methodology to cope with non-stationary disturbances, slowly (and fast changing) RPMs, run-ups and run-downs, etc., is also envisaged as a future demand for this research.

\section{Acknowledgements}

This research is funded by the São Paulo State Research Foundation (FAPESP) - Brazil through grants 2012/157838, and 2014/09894-7, by the National Counsel of Technologic and Scientific Development (CNPq) - Brazil through grant 307369/2013-7, and partially by Flanders Make, the strategic research centre for the manufacturing industry. The authors also acknowledge the support of the KU Leuven research fund.

\section{References}

[1] K. Govindswamy, T. Wellmann, G. Eisele, Aspects of NVH integration in hybrid vehicles, SAE Int. J. Passeng. Cars Mech. Syst. No. 2009-01-2085 (2009) $1-10$.

[2] A. P. Asimakopoulos, B. T. Boumis, C. E. Patsias, D. A. Safacas, E. E. Mitronikas, Experience derived from the conversion of a conventional car to a hybrid electric vehicle - analysis of the powertrain, in: International Symposium on Power Electronics, Electrical Drives, Automation and Motion - SPEEDAM2010, 2010.

[3] J. C. González-Palencia, T. Furubayashi, T. Nakata, Energy use and $\mathrm{CO}_{2}$ emissions reduction potential in passenger car fleet using zero emission vehicles and lightweight materials, Energy 48 (2012) $548-565$.

[4] O. P. Singh, T. Sreenivasulu, M. Kannan, The effect of rubber dampers on engine's NVH and thermal performances, Applied Acoustics 75 (2014) $17-26$.

[5] S. M. Skippon, How consumer drivers construe vehicle performance: implications for electric vehicles, Transportation Research Part F: Traffic Psychology and Behaviour 23 (2014) $15-31$.

[6] R. Guo, C. Cao, Y. Mi, Y. Huang, Experimental investigation of the noise, vibration and harshness performances of a range-extended electric vehicle, Proceedings of the Institution of Mechanical Engineers, Part D: Journal of Automobile Engineering doi: 10.1177/0954407015591329 (2015) $1-14$.

[7] B. James, A. Hoffman, R. W. de Doncker, Reducing noise in an electric vehicle powertrain by means of numerical simulation, Springer International Publishing, 2016, pp. $29-46$.

[8] A. Diez-Ibarbia, M. Battarra, J. Palenzuela, G. Cervantes, S. Walsh, M. de la Cruz, S. Theodossiades, L. Gagliardini, Comparison between transfer path analysis methods on an electric vehicle, Applied Acoustics 118 (2017) $83-101$.

[9] B. K. Sovacool, R. F. Hirsh, Beyond batteries: an examination of the benefits and barriers to plug-in hybrid electric vehicles (PHEVs) and a vehicle-to-grid (V2G) transition, Energy Policy 37 (2009) 1095 - 1103.

[10] C. La, M. Poggi, P. Murphy, O. Zitko, NVH considerations for zero emissions vehicle driveline design, SAE Technical Paper Paper no. 2011-01-1545 (2011) $1-9$

[11] E. Labeye, M. Hugot, C. Brusque, M. A. Regan, The electric vehicle: a new driving experience involving specific skills and rules, Transportation Research Part F: Traffic Psychology and Behaviour 37 (2016) 27 - 40.

[12] H. Campello-Vicente, R. Peral-Orts, N. Campillo-Davo, E. Velasco-Sanchez, The effect of electric vehicles on urban noise maps, Applied Acoustics 116 (2017) $59-64$

[13] C. C. Chan, The state of the art of electric, hybrid, and fuel cell vehicles, Proceedings of the IEEE 95 (2007) $704-718$.

[14] K. Genuit, The future of NVH research - a challenge by new powertrains, SAE Technical Paper No. 2010-36-0515 (2010) 1 - 11.

[15] A. Rust, B. J. Graf, NVH of electric vehicles with range extender, SAE Int. J. Passeng. Cars Mech. Syst. No. 2010-01-1404 (2010) 1 - 8.

[16] S. Varnhagen, A. Same, J. Remillard, J. W. Park, A numerical investigation on the efficiency of range extending systems using advanced vehicle simulator, Journal of Power Sources 196 (2011) 3360 - 3370

[17] D. Schneider, Plug-ins proliferate, IEEE Spectrum - (2012) $36-37$.

[18] D. W. Gao, M. C., A. Emadi, Modeling and simulation of electric and hybrid vehicles, Proceedings of the IEEE 95 (2007) $729-745$.

[19] S. Fujimoto, K. Adachi, H. Ashizawa, J. Motosugi, HEV application of shaking vibration control system based on advanced motor control, SAE Technical Paper Paper no. 2012-01-0622 (2012) 1 - 8.

[20] M. Sarrazin, K. Janssens, H. van der Auweraer, Virtual car sound synthesis technique for brand sound design of hybrid and electric vehicles, SAE Technical Paper Paper no. 2012-36-0614 (2012) 1 - 11.

[21] H. van der Auweraer, D. Sabbatini, E. Sana, K. Janssens, Analysis of electric vehicle noise in view of vulnerable road user safety, in: 18th International Congress on Sound \& Vibration, ICSV18, 2011.

[22] C. J. S. Brizon, E. B. Medeiros, Combining subjective and objective assessments to improve acoustic comfort evaluation of motor cars, Applied Acoustics 73 (2012) 913 - 920.

[23] S. M. Kuo, M. Tahernezhadi, L. Ji, Frequency-domain periodic active noise control and equalization, IEEE Transactions on Speech and Audio Processing 5 (1997) $348-358$. 
[24] M. de Diego, A. Gonzalez, M. Ferrer, G. Piero, Multichannel active noise control system for local spectral reshaping of multifrequency noise, Journal of Sound and Vibration 274 (2004) 249 - 271.

[25] A. Gonzalez, M. de Diego, M. Ferrer, G. Piero, Multichannel active noise equalization of interior noise, IEEE Transactions on Audio, Speech and Language Processing 14 (2006) $110-122$

[26] S. M. Kuo, A. Gupta, S. Mallu, Development of adaptive algorithm for active sound quality control, Journal of Sound and Vibration 299 (2007) $12-21$.

[27] D.-F. Wang, J.-G. Jiang, Z.-W. Liu, X.-L. Cao, Research on ANE algorithm for sound quality control of vehicle interior noise, in: 2010 Third International Conference on Information and Computing, 2010.

[28] A. Gonzalez, M. Ferrer, M. de Diego, G. Piero, J. J. Garcia-Bonito, Sound quality of low-frequency and car engine noises after active noise control, Journal of Sound and Vibration 265 (2003) $663-679$.

[29] L. E. Rees, S. J. Elliott, Adaptive algorithms for active sound-profiling, IEEE Transactions on Audio, Speech and Language Processing 14 (2006) $711-719$.

[30] G. C. Jay, Sound/vibration quality engineering, Sound and vibration 41 (2007) 2 - 9.

[31] H. Fastl, Psycho-acoustics and sound quality, Springer Berlin Heidelberg, Berlin, 2005, pp. 139 - 162.

[32] S. M. Kuo, R. K. Yenduri, A. Gupta, Frequency-domain delayless active sound quality control algorithms, Journal of Sound and Vibration $318(2008) 715-724$

[33] L. P. R. de Oliveira, B. Stallaert, K. Janssens, H. van der Auweraer, P. Sas, W. Desmet., NEX-LMS: A novel adaptive control scheme for harmonic sound quality control, Mechanical Systems and Signal Processing 24 (2010) 1727 - 1738

[34] S.-H. Shin, C. Cheong, Experimental characterization of instrument panel buzz, squeak, and rattle (bsr) in a vehicle, Applied Acoustics 71 (2010) $1162-1168$

[35] Y. S. Wang, G. Q. Shen, H. Guo, X. L. Tang, T. Hamade, Roughness modelling based on human auditory perception for sound quality evaluation of vehicle interior noise, Journal of Sound and Vibration 332 (2013) 3893 - 3904.

[36] J. A. Mosquera-Sánchez, L. P. R. de Oliveira, A multi-harmonic amplitude and relative-phase controller for active sound quality control, Mechanical Systems and Signal Processing 45 (2014) $542-562$.

[37] J. Y. Jeon, J. You, H. Y. Chang, Sound radiation and sound quality characteristics of refrigerator noise in real living environments, Applied Acoustics 68 (2007) $1118-1134$.

[38] S.-K. Lee, Objective evaluation of interior sound quality in passenger cars during acceleration, Journal of Sound and Vibration 310 (2008) $149-168$.

[39] L. P. R. de Oliveira, K. Janssens, P. Gajdatsy, H. van der Auweraer, P. S. Varoto, P. Sas, W. Desmet, Active sound quality control of engine induced cavity noise, Mechanical Systems and Signal Processing 23 (2009) 476 - 488.

[40] A. Nykänen, A. Sirkka, Specification of component sound quality applied to automobile power windows, Applied Acoustics 70 (2009) 813 -820 .

[41] S.-H. Shin, J.-G. Ih, T. Hashimoto, S. Hatano, Sound quality evaluation of the booming sensation for passenger cars, Applied Acoustics 70 (2009) $309-320$

[42] W.-H. Cho, J.-G. Ih, S.-H. Shin, J.-W. Kim, Quality evaluation of car window motors using sound quality metrics, Int. J. Automotive Technology 12 (2011) $443-450$.

[43] G. Tan, D. Wang, S. Chen, J. Song, Active noise control method considering auditory characteristics, SAE Technical Paper Paper no. 2012-01-0993 (2012) $1-6$.

[44] K. Janssens, S. Ahrens, A. Bertrand, J. Lanslots, P. van de Ponselee, A. Vecchio, H. van der Auweraer, An on-line, order-based roughness algorithm, SAE Technical Paper Paper no. 2007-01-2397 (2007) 1 - 11.

[45] S.-K. Lee, H.-W. Kim, E.-W. Na, Improvement of impact noise in a passenger car utilizing sound metric based on wavelet transform, Journal of Sound and Vibration 329 (2010) $3606-3619$.

[46] R. Dragonetti, M. Ponticorvo, P. Dolce, S. di Filippo, F. Mercogliano, Pairwise comparison psychoacoustic test on the noise emitted by DC electrical motors, Applied Acoustics 119 (2017) 108 - 118.

[47] Y. Mollet, M. Sarrazin, H. Van der Auweraer, J. Gyselinck, Experimental noise and vibration analysis of switched reluctance machines - comparison of soft and hard chopping in transient conditions, in: 4th International Conference on Renewable Energy Research and Applications, 2015.

[48] Z. Zhang, M. Shrestha, Sound Quality User-defined Cursor Reading Control - Tonality Metric, Master's thesis, Technical University of Denmark - Brüel \& Kjaer, 2003.

[49] J. A. Mosquera-Sánchez, W. Desmet, L. P. R. de Oliveira, Multichannel feedforward control schemes with coupling compensation for active sound profiling, Journal of Sound and Vibration (2017).

[50] J. A. Mosquera-Sánchez, Controle Ativo Acústico Estrutural: Projeto, Simulação e Análise de Qualidade Sonora (in Portuguese), Master’s thesis, São Carlos School of Engineering - University of São Paulo, 2012.

[51] J. A. Mosquera-Sánchez, W. Desmet, L. P. R. de Oliveira, A multichannel amplitude and relative-phase controller for active sound quality control, Mechanical Systems and Signal Processing 88 (2017) 145 - 165.

[52] H. L. Gilmore, Product conformance cost, Quality Progress 7 (1974) 16 - 19.

[53] D. A. Garvin, What does product quality really mean?, Sloan Management Review 26 (1984) $25-44$.

[54] H. Van der Auweraer, K. Janssens, L. P. R. de Oliveira, M. M. da Silva, W. Desmet, Virtual prototyping for sound quality design of automobiles, Sound \& Vibration 41 (2007) $26-30$

[55] L. P. R. de Oliveira, M. M. da Silva, P. Sas, H. van Brussel, W. Desmet, Concurrent mechatronic design approach for active control of cavity noise, Journal of Sound and Vibration 314 (2008) 507 - 525

[56] H. Fastl, E. Zwicker, Psychoacoustics: Facts and Models, Springer-Verlag, 2007.

[57] A. L. Hastings, Sound Quality of Diesel Engines, Ph.D. thesis, Purdue University, 2004.

[58] P. Daniel, R. Weber, Psychoacoustical roughness: implementation of an optimized model, Acustica - Acta Acustica 83 (1997) 113 - 123.

[59] T. Shin, Y.-J. Lee, S.-K. Lee, A modified tonality and its application to objective evaluation of laser printers noise, Applied Acoustics 79 
(2014) $9-15$.

[60] W. de Baene, A. Vandierendonck, M. Leman, A. Widmann, M. Tervaniemi, Roughness perception in sounds: behavioral and ERP evidence, Biological Psychology 67 (2004) 319 - 330.

[61] L. P. R. de Oliveira, P. S. Varoto, P. Sas, W. Desmet, A state-space modeling approach for active structural acoustic control, Shock and Vibration 16 (2009) $607-621$.

[62] R. Hoeldrich, M. Pflueger, A parametrized model of psychoacoustical roughness for objective vehicle noise quality evaluation, Journal of the Acoustical Society of America 105 (1999) 1371 - 1379.

[63] D. Pressnitzer, S. McAdams, Two phase effects in roughness perception, Journal of the Acoustical Society of America 105 (1999) 2773 2782.

[64] A. Kohlrausch, D. Hermes, R. Duisters, Modeling roughness perception for sounds with ramped and damped temporal envelopes, in: Forum Acusticum, 2005, pp. $1719-1724$.

[65] R. P. Leite, S. Paul, S. N. Y. Gerges, A sound quality-based investigation of the HVAC system noise of an automobile model, Applied Acoustics 70 (2009) $636-645$.

[66] U.-C. Jeong, J.-S. Kim, J.-E. Jeong, I.-H. Yang, J.-E. Oh, Development of a sound quality index for the wash cycle process of front-loading washing machines considering the impacts of individual noise sources, Applied Acoustics 87 (2015) 183 - 189.

[67] Y. S. Wang, C.-M. Lee, D.-G. Kim, Y. Xu, Sound-quality prediction for nonstationary vehicle interior noise based on wavelet pre-processing neural network model, Journal of Sound and Vibration 299 (2007) 933 - 947.

$68]$ G. Pietila, T. C. Lim, Intelligent system approaches to product sound quality evaluations - a review, Applied Acoustics 73 (2012) 987 - 1002.

[69] H. Liu, J. Zhang, P. Guo, F. Bi, H. Yu, G. Ni, Sound quality prediction for engine-radiated noise, Mechanical Systems and Signal Processing $56-57$ (2015) $277-287$.

[70] Y. F. Xing, Y. S. Wang, L. Shi, H. Guo, H. Chen, Sound quality recognition using optimal wavelet-packet transform and artificial neural network methods, Mechanical Systems and Signal Processing 66 - 67 (2016) 875 - 892.

[71] H. B. Huang, R. X. Li, M. L. Yang, T. C. Lim, W. P. Ding, Evaluation of vehicle interior sound quality using a continuous restricted Boltzmann machine-based DBN, Mechanical Systems and Signal Processing 84 (2017) 245 - 267.

[72] F. Campolongo, J. Cariboni, A. Saltelli, An effective screening design analysis for sensitivity analysis of large models, Environmental Modelling \& Software 22 (2007) 1509 - 1518

[73] R. T. Marler, J. S. Arora, Survey of multi-objective optimization methods for engineering, Structural and Multidisciplinary Optimization 26 (2004) $369-395$

[74] K. Deb, Multi-Objective Optimization using Evolutionary Algorithms, John Wiley \& Sons, 2001.

[75] K. Deb, A. Pratap, S. Agarwal, T. Meyarivan, A fast and elitist multiobjective genetic algorithm: NSGA-II, IEEE Transactions on Evolutionary Computation 6 (2002) $182-197$.

[76] E. Zitzler, L. Thiele, Multiobjective evolutionary algorithms: a comparative case study and the strength Pareto approach, IEEE Transactions on Evolutionary Computation 3 (1999) 257 - 271.

[77] E. Zitzler, M. Laumanns, L. Thiele, SPEA2:Improving the strength Pareto evolutionary algorithm, Technical Report, Computer Engineering and Networks Laboratory - Swiss Federal Institute of Technology (ETH) Zurich, 2001.

[78] J. A. Mosquera-Sánchez, Sound quality driven active control of periodic disturbances for hybrid vehicles, Ph.D. thesis, University of São Paulo and Katholieke Universiteit Leuven, 2017.

[79] J. A. Mosquera-Sánchez, K. Janssens, W. Desmet, L. P. R. de Oliveira, Multichannel active sound quality control for independent-channel sound profiling, in: Euronoise2015, 2015.

[80] MathWorks., Global optimization toolbox: User's guide (r2016a), Website, 2016. URL: https://www.mathworks.com/help/optim/ index.html.

[81] L. P. R. de Oliveira, M. M. da Silva, J. A. Mosquera-Sánchez, L. A. M. Gonalves, Loudness scattering due to vibro-acoustic model variability, Journal of the Brazilian Society of Mechanical Sciences \& Engineering 34 (2012) 604 - 611.

[82] S. M. Kuo, D. R. Morgan, Active Noise Control Systems: algorithms and DSP implementations, Wiley, 1996.

[83] L. Vicente, E. Masgrau, Novel FxLMS convergence condition with deterministic reference, IEEE Transactions on Signal Processing 54 (2006) 3768 - 3774.

[84] H. Vold, M. Mains, J. Blough, Theoretical foundations for high performance order tracking with the Vold-Kalman tracking filter, SAE Technical Paper Paper no. 972007 (1997) 1 - 6. 